\title{
Unitary Spherical Spectrum for p-Adic Classical Groups
}

\author{
DAN BARBASCH \\ Department of Mathematics, Cornell University, Ithaca, NY 14853, U.S.A. \\ e-mail: barbasch@polygon.math.cornell.edu
}

\begin{abstract}
ALLEN MOY
Department of Mathematics, University of Michigan, Ann Arbor, MI 4822I, U.S.A. e-mail: moy@math.lsa.umich.edu
\end{abstract}

(Received: 26 October 1995)

\begin{abstract}
Let $G$ be a split reductive p-adic group. Then the determination of the unitary representations with nontrivial Iwahori fixed vectors can be reduced to the determination of the unitary dual of the corresponding Iwahori-Hecke algebra. In this paper we study the unitary dual of the Iwahori-Hecke algebras corresponding to the classical groups. We determine all the unitary spherical representations.
\end{abstract}

Mathematics Subject Classifications (1991). 22E50, 16-XX, 20-XX.

Key words: unitary dual, p-adic groups, Iwahori-Hecke algebra.

\section{Introduction}

The purpose of this paper is to describe the spherical unitary dual for the classical split groups. The simplest reasonable statement that might be true about the spherical dual would be the following:

A spherical irreducible representation is unitary if and only if it is a complementary series from an anti-tempered representation $(\mathcal{I M}$ of a tempered representation).

Unfortunately this is not quite so simple; there are complementary series induced from complementary series for $\mathrm{Gl}(n)$ such that the nearest parameter which is induced from a tempered representation is reducible.

The paper deals mainly with types B, C and D since the (general) case of $\mathrm{Gl}(n)$ is already done in [11]. The first section reviews general results on the representations of p-adic groups and their relation to the representation theory of Hecke algebras. In particular we reduce the problem to an equivalent one on the graded algebra introduced by Lusztig. In this context, we study the classification of the irreducible Hermitian modules in detail. The setting is almost exclusively that of the graded Hecke algebra, we have tried to use as little as possible from the Langlands classification for p-adic groups. In fact, there are only two places 
where we use the theory on the group. The first is to show that there exists a nowhere zero intertwining operator (at the end of 1.7, but this is not really used for classifying the unitary spectrum). The second is the fact that certain modules of the Hecke algebra (Sections 2.1 and 2.2) are unitary.

In Sections 2.3 and 2.4, we refine a nonunitarity criterion first used by Casselman. This is our main tool for ruling out the parameters that are not unitary. The phrasing is reminiscent of the Dirac inequality or the use of K-types in $\mathfrak{p}$ in the case of real groups.

In Section 3, we write down an explicit parametrization of the Hermitian spherical modules. We use this in Section 4 where we prove the main results. The crucial ones are in 4.2 and 4.3. The basic ideas of the arguments are fairly simple in nature. However, they are quite delicate technically, and require some ingenuity. We do not give complete proofs in all cases, but rather treat representative cases in detail. Many of the results are more general (nonspherical parameters, Hecke algebras with unequal parameters), but it is not quite clear at this writing how far they can be pushed. Certainly the arguments work in the case of Langlands parameters coming from maximal parabolic subgroups. Via the Iwahori-Matsumoto involution these cases contain the spherical cases. It is in this setting that we prove our results.

\section{Preliminaries}

\subsection{UNRAMIFIED REPRESENTATIONS}

Let $\mathbb{F}$ be a p-adic field. Denote by $G$ be the $\mathbb{F}$-rational points of a linear algebraic reductive group defined over $\mathbb{F}$. We assume that $G$ is split. Denote by $A$ a maximally split torus and fix a Borel subgroup with decomposition $B=A N$.

PROBLEM. Let $\nu \in \hat{A}$ be an unramified character and $I(\nu)$ be the unramified principal series. Then it is important to study the composition series and irreducible subquotients of $I(\nu)$. This is done via the Hecke algebra attached to the Iwahori subgroup. Let

$$
\begin{aligned}
& \mathcal{R}=\{x \in \mathbb{F}:|x| \leqslant 1\}, \quad \mathcal{P}=\{x \in \mathbb{F}:|x|<1\}, \\
& \Omega=\{x \in \mathbb{F}:|x|=1\} .
\end{aligned}
$$

Then $G$ has a maximal compact subgroup $K=G(\mathcal{R})$, and there is an exact sequence

$$
1 \longrightarrow K_{1} \longrightarrow K \longrightarrow G(\mathcal{R} / \mathcal{P}) \cong G\left(\mathbb{F}_{q}\right) \longrightarrow 1 .
$$

Fix a Borel subgroup in $G\left(\mathbb{F}_{q}\right)$. Then its inverse image is an open compact subgroup called the Iwahori subgroup and denoted $\mathcal{I}$. The algebra of compact 
supported bi-invariant functions $\mathcal{H}:=\mathcal{H}(\mathcal{I} \backslash G / \mathcal{I})$ is called the Hecke algebra. Let

$$
\begin{aligned}
\mathcal{C}(\mathcal{I})= & \text { the category of admissible finite length representations } \\
& \text { so that all their subquotients are } \\
& \text { generated by their Iwahori fixed vectors. }
\end{aligned}
$$

THEOREM ([1]). The functor $V \mapsto V^{\mathcal{I}}$ is an equivalence of categories from $\mathcal{C}(\mathcal{I})$ to $\mathcal{C}(\mathcal{H})$, the category of finite-dimensional representations of $\mathcal{H}$. The inverse is given by $W \mapsto \mathcal{H}(G / \mathcal{I}) \otimes W$.

An irreducible representation $\pi$ is in $\mathcal{C}(\mathcal{I})$ iff it is a subquotient of an $I(\nu)$.

$\mathcal{H}$ also has a star operation given by

$$
f^{*}(x)=\overline{f\left(x^{-1}\right)}
$$

so we can talk about Hermitian and unitary modules. It is more or less clear that an admissible representation $V \in \mathcal{C}(\mathcal{I})$ of $G$ is Hermitian iff $V^{\mathcal{I}}$ is Hermitian. It is also clear that if $V$ is unitary, then so is $V^{\mathcal{I}}$. The converse is the subject of two papers [2] and [3]. The result is summarized in the next theorem.

THEOREM. An irreducible representation in $\mathcal{C}(\mathcal{I})$ is unitary if and only if $V^{\mathcal{I}}$ is unitary.

\subsection{THE GRADED HECKE ALGEBRA}

The Hecke algebra $\mathcal{H}$ can be described by generators and relations. Let $z$ be an indeterminate (which can then be specialized to $q^{1 / 2}$ ). Let $\Pi \subset R^{+} \subset R$ be the simple roots, positive roots and roots corresponding to $A \subset B$, and $S$ be the simple root reflections. Let $\mathcal{X}=\check{\mathcal{Y}}=\operatorname{Hom}\left(G_{m}, A\right)$ be the algebraic homomorphisms of $G_{m}=\mathrm{Gl}(1, \mathbb{F})$ and $\mathcal{Y}=\check{\mathcal{X}}=\operatorname{Hom}\left(A, G_{m}\right)$. Then $\mathcal{H}$ can be characterized as the Hecke algebra over $\mathbb{C}\left[z, z^{-1}\right]$ attached to the root datum $\mathcal{R}=(\mathcal{Y}, \mathcal{X}, \check{R}, R, \check{\Pi})$. The set of generators we will use is the one first introduced by Bernstein. Denote by ${ }^{L_{G}} G$ the (complex) dual group, with maximal torus ${ }^{L_{T}}$ and Borel subgroup ${ }^{L_{B}} B$ containing ${ }^{L} T$. Let

$$
\mathcal{A}=\text { rational functions on } \mathbb{C}^{*} \times{ }^{L} T \text {. }
$$

Then $\mathcal{H}$ is generated by $\left\{T_{w}\right\}_{w \in W}$ and $\left\{\theta_{x}\right\}_{x \in \mathcal{X}}$ subject to the relations

$$
\begin{aligned}
& T_{w} T_{w^{\prime}}=T_{w w^{\prime}} \quad\left(l(w)+l\left(w^{\prime}\right)=l\left(w w^{\prime}\right)\right), \\
& T_{s}^{2}=\left(z^{2}-1\right) T_{s}+z^{2}, \quad \theta_{x} T_{s}=T_{s} \theta_{s x}+\left(z^{2}-1\right) \frac{\theta_{x}-\theta_{s x}}{1-\theta_{\alpha}} .
\end{aligned}
$$

This realization is very convenient for detemining the center of $\mathcal{H}$ and, thus, computing infinitesimal characters of representations. 
THEOREM (Bernstein-Lusztig). The center of $\mathcal{H}$ is given by the Weyl group invariants in $\mathcal{A}$.

In particular, infinitesimal characters are parametrized by $W$-orbits $\chi=(q, t) \in$ $\mathbb{C}^{*} \times{ }^{L_{T}}$. (We always assume that $q$ is real or at least not a root of unity.) In particular, such an infinitesimal character is called real if $s$ has no elliptic part.

The study can be simplified significantly by using the graded Hecke algebra introduced by Lusztig. Let

$$
\mathcal{J}=\{f \in \mathcal{A}: f(1,1)=0\} .
$$

This ideal satisfies $\mathcal{H} \mathcal{J}=\mathcal{J H}$, so we can introduce a filtration

$$
\mathcal{H}=\mathcal{H}^{0} \supset \cdots \supset \mathcal{H}^{i} \supset \mathcal{H}^{i+1} \supset \cdots,
$$

and form the graded object $\mathbb{H}$. It can be written as

$$
\mathbb{H}=\mathbb{C}[\mathbf{r}] W \times \mathbb{A},
$$

where $\mathbf{r} \equiv z-1(\bmod \mathcal{J})$, and $\mathbb{A}$ is the symmetric algebra over $L_{\mathfrak{t}}=\mathcal{X} \otimes_{\mathbb{Z}} \mathbb{C}$. The previous relations become

$t_{w} t_{w^{\prime}}=t_{w w^{\prime}}, \quad t_{s}^{2}=1, \quad t_{s} \omega=s(\omega) t_{s}+2 \mathbf{r}\langle\omega, \check{\alpha}\rangle \quad\left(s=s_{\alpha}\right)$.

The center of $\mathbb{H}$ is $\mathbb{A}^{W}$. In particular, infinitesimal characters are parametrized by $W$-orbits of elements $\bar{\chi}=(r, s) \in \mathbb{C} \times{ }^{L} \mathfrak{t}$.

THEOREM ([8]). There is a matching $\chi \leftrightarrow \bar{\chi}$ between real infinitesimal characters $\chi$ of $\mathcal{H}$ and infinitesimal characters $\bar{\chi}$ of $\mathbb{H}$ so that if $\mathcal{H}_{\chi}$ and $\mathbb{H} \bar{\chi}$ are the quotients by the corresponding ideals, then $\mathcal{H}_{\chi} \cong \mathbb{H} \bar{\chi}$.

Remark. We (fix a real $r \neq 0$ and) transfer the study of the representation theory of $\mathcal{H}$ to $\mathbb{H}$. The classification of irreducible representations is as according to [6] and [10]; irreducible representations are parametrized by ${ }^{L} G$ conjugacy classes $(s, e, \psi)$, where $s \in{ }^{L} G$ is semisimple, $e \in{ }^{L} \mathfrak{g}$ is nilpotent such that $\operatorname{Ad}(s) e=r e$ and $\psi \in A(\widehat{s, e)}$ is an irreducible representation of the component group of the centralizer of $s$ and $e$. We also recall that [6] attaches to each such parameter a standard module $X(s, e, \psi)$. Then the results in [2] imply that each $X(s, e, \psi)$ contains a lowest $\mathrm{K}$-type occuring with multiplicity 1 given by the Springer correspondence. Furthermore, the group $A(e) / A(s, e)$ plays the role of an $R$-group.

\subsection{HERMITIAN MODULES}

The * operation also transfers to the graded version. We refer to Section 5 of [3] for the details. Here is a summary of what we need. Let $w_{0} \in W$ be the longest 
element, $t_{0}$ be the corresponding element in $\mathbb{C} W$. Since ${ }_{\mathfrak{t}}=\mathcal{X} \otimes_{\mathbb{Z}} \mathbb{C}$, it has a conjugation coming from the complex conjugation on $\mathbb{C}$. We denote it by ${ }^{-}$. Let $\iota(\omega)=(-1)^{\operatorname{deg} \omega} \bar{\omega}$ and $\tilde{\omega}=w_{0} \iota(\omega)$.

THEOREM (Section 5 in [3]). Let $\omega \in \mathbb{A}$. Then $t_{w}^{*}=t_{w^{-1}}, \omega^{*}=t_{0} \cdot \tilde{\omega} \cdot t_{0}$. In particular, if $\omega \in{ }^{L_{\mathfrak{t}}}$, then

$$
\omega^{*}=-\bar{\omega}+2 r \sum_{\beta \in R^{+}}\langle\bar{\omega}, \check{\beta}\rangle t(\beta)
$$

where $t(\beta) \in \mathbb{C}[W]$ is the reflection about $\beta$.

The main result of [3] can be summarized as follows. Let $(e, H, f)$ be a Lie triple corresponding to $e$. Write $s=s_{0} s_{H}$, where $s_{0}$ is an element centralizing the triple and $s_{H}=\exp (1 / 2 \log q H)$. Let $s_{0}=s_{e} s_{h}$ be the decomposition of $s_{0}$ into elliptic and hyperbolic parts. Denote by $\bar{s}=s_{e} s_{h}^{-1}$. An irreducible representation admits a Hermitian form if and only if $(s, e, \psi)$ is conjugate to $\left(s^{\prime}=\bar{s}_{0} s_{H}, e, \psi\right)$. A parameter will be called real if $s$ has trivial elliptic part.

COROLLARY. The classification of the unitary dual of a split p-adic group is the same as the classification of the unitary dual of the corresponding graded Hecke algebra. Furthermore, using certain isomorphisms of these algebras it is sufficient to consider the case of real parameter.

\subsection{HERMITIAN DUALS}

Fix a (standard) parabolic subgroup $P$ with Levi decomposition $P=M N$. Let $\mathbb{H}_{M}$ be the corresponding (graded) Hecke subalgebra. Every element $a \in \mathbb{H}$ can be written uniquely as $a=\sum_{w \in W / W(M)} t_{w} m_{w}$ with $m_{w} \in \mathbb{H}_{M}$. Thus, there is a well defined map

$$
\varepsilon_{M}: \mathbb{H} \rightarrow \mathbb{H}_{M}, \quad \varepsilon_{M}(a):=m_{1}, \text { the component of } a \text { in } \mathbb{H}_{M} .
$$

PROPOSITION. Denote by $*_{M}$ the map corresponding to the star operation $*_{M}: a \mapsto a^{*}$ in $\mathbb{H}_{M}$ and denote by $*_{G}$ the corresponding map in $\mathbb{H}$. Then $\varepsilon_{M}\left(*_{G} a\right)=*_{M} \varepsilon_{M}(a)$, for all $a \in \mathbb{H}$.

Proof. First observe that

$$
\varepsilon_{M}\left(t_{m_{1}} a t_{m_{2}}\right)=t_{m_{1}} \varepsilon(a) t_{m_{2}}, \quad \text { for } m_{i} \in W(M) .
$$

Let $\omega \in \mathbb{A}$. The long element $w_{0}$ decomposes as $w_{0}=w_{l} w_{0}^{M}$, where $w_{0}^{M}$ is the long element in $W(M)$. Let $t_{0}, t_{l}$ and $t_{0}^{M}$ be the corresponding elements in $\mathbb{H}$. Since $t_{0}^{-1}=t_{0}$ and $\left(t_{0}^{M}\right)^{-1}=t_{0}^{M}$, the relation

$$
t_{0}=t_{l} t_{0}^{M}=t_{0}^{M} t_{l}^{-1}
$$


holds. Then we get

$$
*_{G}(\omega)=t_{0} \cdot \tilde{\omega} \cdot t_{0}=t_{l} t_{0}^{M} \cdot w_{0}^{M} w_{l}^{-1}(\iota(\omega)) \cdot t_{l} t_{0}^{M} .
$$

On the other hand,

$$
\begin{aligned}
w_{0}^{M} w_{l}^{-1} \iota(\omega) \cdot t_{l} & =t_{l} \cdot w_{l}^{-1} w_{0}^{M} w_{l}^{-1} \iota(\omega)+\sum_{x<w_{l}} t_{x} \omega_{x} \\
& =t_{l} \cdot w_{0}^{M} \iota(\omega)+\sum_{x<w_{l}} t_{x} \omega_{x},
\end{aligned}
$$

where the sum ranges over $x \in W$ which are smaller than $w_{l}$ in the Bruhat order. Combining this with (1.4.4) and the formula for $*_{M}$ in Theorem 1.3, we get

$$
*_{G}(\omega)=*_{M}(\omega)+\sum_{x<w_{l}} t_{l} t_{0}^{M} t_{x} \omega_{x} t_{0}^{M} .
$$

Therefore, using (1.4.2),

$$
\begin{aligned}
\varepsilon\left(*_{G}(\omega)\right) & =*_{M}(\omega)+t_{0}^{M} \varepsilon\left(t_{l}^{-1} t_{x}\right) \omega_{x} t_{0}^{M} \\
& =*_{M}(\omega)+t_{0}^{M} \varepsilon\left(t_{w_{l}^{-1} x}\right) \omega_{x} t_{0}^{M} .
\end{aligned}
$$

To prove the formula in the proposition for $a=\omega$, we must show that $\varepsilon\left(t_{w_{l}^{-1} x}\right)=$ 0 for $x<w_{l}$. But if $w_{l}^{-1} x=m \in W(M)$, then $w_{l}=x m^{-1}$ with $x<w_{l}$, contradicting the minimality property of $w_{l}$.

Now consider an element $t_{w} \omega$ such that $w \notin W(M)$. Then $\varepsilon_{M}\left(t_{w} \omega\right)=0$. We need to show that $\varepsilon_{M}\left(*_{G}(\omega) t_{w^{-1}}\right)=0$ as well. For this, write $*_{G}(\omega)=t_{0} \cdot \tilde{\omega} \cdot t_{0}$ and decompose

$$
t_{0} \cdot \tilde{\omega} \cdot t_{0} \cdot t_{w^{-1}}=t_{0} \cdot \tilde{\omega} \cdot t_{w_{0} w^{-1}}=\sum_{x<w_{0} w^{-1}} t_{w_{0} x} \omega_{x}, \quad \omega_{x} \in \mathbb{A} .
$$

To prove the claim we need to show $w_{0} x \notin W(M)$. Suppose $w_{0} x=m$. Then $x=w_{0} m$, and so $w_{0} m<w_{0} w^{-1}$. It follows that $w_{0} w^{-1} \in w_{0} W(M)$, because $w_{0}$ is the long element. But this implies $w^{-1} \in W(M)$, a contradiction. Thus $\varepsilon_{M}\left(*_{G}(\omega) t_{w^{-1}}\right)=0$ as claimed.

If $\mathcal{W}$ is a module for $\mathbb{H}_{M}$, then we can form the induced module

$$
I(\mathcal{W}):=\mathbb{H} \otimes_{\mathbb{H}_{M}} \mathcal{W}
$$

This has a basis $\left\{t_{x} \otimes v\right\}$ where $v \in \mathcal{W}$ and $x \in W / W(M)$.

COROLLARY. The Hermitian dual of $I(\mathcal{W})$ is $I\left(\mathcal{W}^{h}\right)=\mathbb{H} \otimes_{\mathbb{H}_{M}} \mathcal{W}^{h}$. More precisely, let $t_{x} \otimes v_{x} \in I(\mathcal{W}), t_{y} \otimes v_{y} \in I\left(\mathcal{W}^{h}\right)$, and $\langle,\rangle_{M}$ be the pairing of $\mathcal{W}$ with $\mathcal{W}^{h}$. Then the pairing between $I(\mathcal{W})$ and $I\left(\mathcal{W}^{h}\right)$ is given by

$$
\left\langle t_{x} \otimes v_{x}, t_{y} \otimes v_{y}\right\rangle:=\left\langle\varepsilon_{M}\left(t_{y}^{*} t_{x}\right) v_{x}, v_{y}\right\rangle_{M}
$$


Proof. First observe that these spaces are finite-dimensional, so it is sufficient to construct an injection

$$
\mathbb{H} \otimes_{\mathbb{H}_{M}} \mathcal{W}^{h} \longrightarrow\left[\mathbb{H} \otimes_{\mathbb{H}_{M}} \mathcal{W}\right]^{h} \text {. }
$$

The pairing $\langle$,$\rangle gives such an injection with the required properties by Propo-$ sition 1.4 .

\subsection{LANGLANDS CLASSIFICATION}

Suppose $a$ is an automorphism between two root data $\mathcal{R}_{1}$ and $\mathcal{R}_{2}$. This induces an automorphism of the corresponding Hecke algebras,

$$
\text { a: } \mathbb{H}_{2} \longrightarrow \mathbb{H}_{1} \text {. }
$$

In particular, let $M_{1}, M_{2}$ be two Levi components of standard parabolic subgroups. Suppose $w \in W$ satisfies $w M_{1} w^{-1}=M_{2}$ and is minimal in its double coset $W\left(M_{2}\right) w W\left(M_{1}\right)$. Then the above discussion gives an isomorphism

$$
a_{w}: \mathbb{H}_{M_{2}} \longrightarrow \mathbb{H}_{M_{1}}
$$

which we use to transfer representations $\mathcal{V}$ of $\mathbb{H}_{M_{1}}$ to $w \mathcal{V}$ of $\mathbb{H}_{M_{2}}$.

Recall that according to the (classical version of the) Langlands classification, every irreducible module can be realized as the unique irreducible quotient $L(M, \mathcal{W}, \nu)$ of a standard module

$$
X(M, \mathcal{W}, \nu):=\mathbb{H} \otimes_{\mathbb{H}_{M}}\left[\mathcal{W} \otimes \mathbb{I}_{\nu}\right],
$$

where

$\mathcal{W}$ is tempered irreducible and $\langle\operatorname{Re} \nu, \check{\alpha}\rangle>0$,

$$
\text { for all } \alpha \in \Delta(\mathfrak{n}), \mathfrak{n}:=\operatorname{Lie}(N) \text {. }
$$

The module $X(M, \mathcal{W}, \nu)$ of course coincides with $I\left(M, \mathcal{W} \otimes \mathbb{I}_{\nu}\right)$, but we use the notation (1.5.3) to emphasize that it satisfies (1.5.4). Two Langlands quotients $L(M, \mathcal{W}, \nu)$ and $L\left(M^{\prime}, \mathcal{W}^{\prime}, \nu^{\prime}\right)$ are isomorphic if and only if there is $w \in W$ such that

$$
w(M, \mathcal{W}, \nu)=\left(M^{\prime}, \mathcal{W}^{\prime}, \nu^{\prime}\right) .
$$

If on the other hand $\langle\operatorname{Re} \nu, \check{\alpha}\rangle<0$, then the module $I\left(M, \mathcal{W} \otimes \mathbf{I}_{\nu}\right)$ has a unique irreducible submodule, namely $L\left(w_{m} M, w_{m} \mathcal{W}, w_{m} \nu\right)$, where $w_{m}$ be the minimal element in $W\left(w_{0} M\right) w_{0} W(M)$.

PROPOSITION. The Hermitian dual of $L(M, \mathcal{W}, \nu)$ is $L\left(w_{0} M, w_{m} \mathcal{W},-w_{0} \bar{\nu}\right)$. In particular, $L$ is Hermitian if and only if there is an element $w$ such that $w \cdot(M, \mathcal{W}, \nu)=(M, \mathcal{W},-\bar{\nu})$ 
Proof. The Hermitian dual of $X(M, \mathcal{W}, \nu)$ is $I(M, \mathcal{W},-\bar{\nu})$. This is not a standard module because $-\bar{\nu}$ fails to satisfy (1.5.4). However, $\langle-\operatorname{Re} \bar{\nu}, \check{\alpha}\rangle\langle 0$, so $I(M, \mathcal{W},-\bar{\nu})$ has $L\left(w_{0} M, w_{m} \mathcal{W},-w_{0} \bar{\nu}\right)$ as unique irreducible submodule. Thus

$$
L(M, \mathcal{W}, \nu)^{h} \cong L\left(w_{0} M, w_{m} \mathcal{W},-w_{0} \bar{\nu}\right)
$$

as claimed.

\subsection{INTERTWINING OPERATORS}

We use the notation of 1.5. Suppose that $w M_{1} w^{-1}=M_{2}$ is minimal in its double coset $W\left(M_{2}\right) w W\left(M_{1}\right)$ (or rather the ${ }^{L} M$ 's).

Given a simple reflection $s_{\alpha}$, we can form the element $r_{\alpha} \in \mathbb{H}$,

$$
r_{\alpha}:=t_{\alpha} \alpha-2 r \text {. }
$$

LEMMA. The elements $r_{\alpha}$ satisfy $\omega r_{\alpha}=r_{\alpha} s(\omega)$. Let $w=s_{1} \cdots s_{k}$ be a reduced decomposition. Then $r_{w}=\prod r_{i}$ is well defined.

Proof. The first relation is a simple application of the defining relations for $\mathbb{H}$ :

$$
\begin{aligned}
\omega\left(t_{\alpha} \alpha-2 r\right) & =\left(t_{\alpha} s(\omega)+2 r\langle\omega, \check{\alpha}\rangle\right) \alpha-2 r \omega \\
& =\left(t_{\alpha} \alpha-2 r\right) s(\omega)-2 r(s(\omega)-\omega)+2 r\langle\omega, \check{\alpha}\rangle \alpha \\
& =\left(t_{\alpha} \alpha-2 r\right) s(\omega) .
\end{aligned}
$$

More generally, if $f$ is a rational function in one variable, then $f(\omega) r_{\alpha}=$ $r_{\alpha} f\left(s_{\alpha} \omega\right)$.

Recall from [7] that

$$
\bar{\tau}_{\alpha}=t_{\alpha} \frac{\alpha}{\alpha+2 r}-\frac{2 r}{\alpha+2 r}=\frac{1}{-\alpha+2 r} r_{\alpha},
$$

and that $\bar{\tau}_{w}=\prod \bar{\tau}_{i}$ is well defined (i.e., independent of the reduced decomposition). Write

$$
R_{w}=\left\{\alpha_{1}, s_{1} \alpha_{2}, \ldots, s_{1} s_{2} \cdots s_{k-1} \alpha_{k}\right\}=\left\{\beta>0: w^{-1} \beta<0\right\} .
$$

Then substituting (1.6.3) for each $r_{i}$ and applying (1.6.2) repeatedly, we get

$$
\begin{aligned}
\prod r_{i} & =\prod\left(-\alpha_{i}+2 r\right) \bar{\tau}_{i}=\left(\prod_{\beta \in R_{w}}(-\beta+2 r)\right) \prod \bar{\tau}_{i} \\
& =\left(\prod_{\beta \in R_{w}}(-\beta+2 r)\right) \bar{\tau}_{w} .
\end{aligned}
$$

The claim follows. 
PROPOSITION. Let $\mathcal{V}$ be a representation of $\mathbb{H}_{M_{1}}$. Then the operator $A_{w}$ : $I\left(M_{1}, \mathcal{V}\right) \rightarrow I\left(M_{2}, w \mathcal{V}\right)$ defined by $A_{w}\left(t_{x} \otimes_{\mathbb{H}_{M_{1}}} v\right):=t_{x} r_{w} \otimes_{\mathbb{H}_{M_{2}}} v$ is an intertwining operator.

Proof. We need to check that $A_{w}$ is well defined, i.e., it satisfies

$$
\begin{aligned}
& \omega r_{w}=r_{w} w^{-1}(\omega), \quad t_{\alpha} r_{w}=r_{w} t_{w^{-1} \alpha}, \\
& \alpha \in \Delta\left(M_{1}\right) \text { a simple root. }
\end{aligned}
$$

The first relation follows from a repeated application of Lemma 1.6. For the second relation, $r_{\alpha} r_{w}=r_{w} r_{w^{-1} \alpha}$ holds. Substituting $r_{\alpha}=t_{\alpha} \alpha-2 r$ and using $\alpha r_{w}=r_{w} w^{-1} \alpha$, we get the second relation. The fact that the map is an intertwining operator follows from the nature of the action of $\mathbb{H}$ which is by multiplication on the left.

\subsection{INTERTWINING OPERATORS FOR STANDARD MODULES}

We apply the results in 1.6 to the Langlands classification. Recall $w_{m}$ the minimal element in the double coset $W(M) w_{0} W\left(w_{0} M\right)$. Then multiplication on the right by $r_{m}:=r_{w_{m}}$ is an intertwining operator

$$
A_{m}: X(M, \mathcal{W}, \nu) \longrightarrow I\left(w_{0} M, w_{m} \mathcal{W}, w_{0} \nu\right)
$$

This is identically zero precisely when $r_{m} \otimes\left[v \mathbb{I}_{\nu}\right]=0$ for all $v \in \mathcal{W}$. When it is not identically zero, the image is precisely $L(M, \mathcal{W}, \nu)$ because this submodule

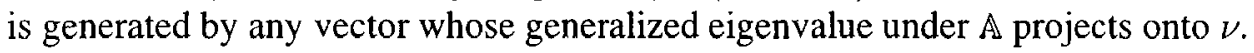
On the other hand, observe that the leading term of $r_{m}$ is $t_{m} \prod_{(\alpha, \nu)>0} \alpha$. But

$$
\left(\prod_{(\alpha, \nu)>0} \alpha\right) \cdot v \mathbb{\pi}_{\nu}=\prod_{(\alpha, \nu)>0}(\alpha, \nu+\chi) v \mathbf{I}_{\nu},
$$

where $\chi$ is the infinitesimal character of $\mathcal{W}$. This is because $\prod_{(\alpha, \nu)>0} \alpha$ is invariant under $W(M)$ therefore in the center of $\mathbb{H}_{M}$. Thus $A_{m}$ is not identically zero as a function of $\nu$. For a full principal series (1.7.2) implies that $A_{m}$ is not identically zero for any $\nu$ satisfying (1.5.4). But for more general induced representations $A_{m}$ could be zero for certain values of $\nu$. On the other hand, it follows from the theory of intertwining operators on the p-adic group, that there exists a family of intertwining operators $B_{m}(\nu)$

$$
B_{m}: X(M, \mathcal{W}, \nu) \longrightarrow I\left(w_{m} M, w_{m} \mathcal{W}, w_{m} \nu\right)
$$

which is analytic as a function of $\nu$ and whose image is $L(M, \mathcal{W}, \nu)$ for all $\nu$ satisfying (1.5.4). We claim that there is a meromorphic function $f(\nu)$ such that $f(\nu) A_{m}=B_{m}(\nu)$. This goes as follows. Recall that $X$ has a lowest K-type $\mu$ occurring with multiplicity 1 . Then there are analytic functions $g$ and $h$ such that 
for any vector $v \in X$ transforming according to $\mu$, we have $A_{m}(v)=g(\nu) v$ and $B_{m} v=h(\nu) v$. Then $f=h g^{-1}$.

We will not need this refinement.

\subsection{HERMITIAN FORMS}

Suppose $L(M, \mathcal{W}, \nu)$ is Hermitian with $\nu$ real. Let $w$ be such that $w(M, \mathcal{W}, \nu)=$ $(M, \mathcal{W},-\nu)$, minimal in its double coset $W(M) w W(M)$. This double coset is also $W(M) w_{0} W(M)$. This is because both $w \nu$ and $w_{0} \nu$ are antidominant (so $\left.w_{0} \nu=w \nu=-\nu\right)$ and $\Delta(M)=\{\alpha \in \Delta:(\alpha, \nu)=0\}$. Denote by $a_{w}$ the isomorphism (1.5.2) and by $\tau: \mathcal{W} \rightarrow \mathcal{W}$ an isomorphism (unique up to a scalar) satisfying $\tau(h \cdot v)=a_{w}(h) \cdot \tau(v)$. Recall that we can decompose $M={ }^{0} M \cdot A$, where $A$ is the (split part of the) center of $M$. Then we can write any element $m \in \mathbb{H}_{M}$ as $m=\sum m_{i} a_{i}$ with $m_{i} \in \mathbb{H}^{\prime}{ }_{M}$ and $a_{i} \in A$. Thus, it makes sense to evaluate $m \in \mathbb{H}_{M}$ at $\nu$. We write this as $m(\nu)$. With this notation

$$
\sum m_{i} a_{i}\left(v \mathbb{I}_{\nu}\right)=\sum m_{i} a_{i}(\nu) v \mathbb{I}_{v}=(m(\nu) v) \mathbb{I}_{\nu}
$$

COROLLARY. Suppose $X(M, \mathcal{W}, \nu)$ is Hermitian irreducible and $\nu$ is real. Then up to a nonzero scalar the inner product is given by the formula

$$
\left\langle t_{x} \otimes v_{x} \mathbb{I}_{\nu}, t_{y} \otimes v_{y} \mathbb{I}_{\nu}\right\rangle=\left\langle\varepsilon\left(t_{y}^{*} t_{x} r_{w}\right)(\nu) \tau\left(v_{x}\right), v_{y}\right\rangle_{\mathcal{W}} .
$$

Proof. This follows from 1.4, 1.6 and 1.7.

There are two main cases that we will consider.

EXAMPLE 1. Suppose $M=\mathrm{Gl}(k) \times \mathrm{Gl}(m) \times \mathrm{Gl}(k) \subset G=\mathrm{Gl}(2 k+m)$ and $\mathcal{W}=\mathrm{St} \otimes \mathcal{W}_{0} \otimes S t$, where St stands for the Steinberg representation. The element $w$ interchanges the $\mathrm{Gl}(k)$-factors. In this case we can take $\tau=\mathrm{Id}$. This is because since $\operatorname{St}\left(t_{\alpha}\right) v=-v$ and $\operatorname{St}(\alpha) v=-2 r v$ for all simple roots $\alpha$, and so

$$
\mathrm{St}\left(m_{1}\right) v_{1} \otimes \mathrm{St}\left(m_{2}\right) v_{2}=\mathrm{St}\left(m_{2}\right) v_{1} \otimes \mathrm{St}\left(m_{1}\right) v_{2}
$$

EXAMPLE 2. Suppose $M=\mathrm{Gl}(k) \times G(m) \subset G(m+k)$ where $G(n)$ is a classical group of rank $n$ other than $\mathrm{Gl}(n)$. Then $\mathcal{W}=\mathrm{St} \otimes \mathcal{W}_{0}$. The minimal element $w_{m}$ induces the outer automorphism on $\mathrm{Gl}(k)$ and the identity on $G(m)$ if $G(m)$ is of type B or C; due to the properties of St, we can take $\tau=\mathrm{Id}$. In type $\mathrm{D}$, if $k$ is odd, the representation $\mathcal{W}_{0}$ must be equivalent to the one obtained by composing with the automorphism of order two permuting the last two roots in the diagram. We write $\tau$ for the intertwining operator of $\mathcal{W}_{0}$. 


\section{A Unitarity Criterion}

\subsection{CHARACTER THEORY}

We review some material from [2]. Recall the existence of the Iwahori-Matsumoto involution $\mathcal{I} \mathcal{M}$ on the Hecke algebra,

$$
\mathcal{I} \mathcal{M}\left(t_{w}\right)=(-1)^{l(w)} t_{w}, \quad \mathcal{I} \mathcal{M}(\omega)=-\omega, \quad \omega \in{ }^{L_{t}}
$$

Given the infinitesimal character $\chi$, there is a unique irreducible $L(\chi ; \operatorname{sgn})$ (containing sgn with multiplicity 1 ); and $\mathcal{I M}$ takes it to a spherical module. This is due to the form of $\mathcal{I M}$ on $W$. The classification theorem of representations of $\mathbb{H}$ then gives the following result.

THEOREM ([2]). Let $\chi$ be an infinitesimal character corresponding to the semisimple element $h \in{ }^{L_{\mathfrak{g}}}$. Then there is a unique orbit of maximal dimension (with representative $\left.e_{\max }\right)$ such that $\left[h, e_{\max }\right]=2 e_{\max }$. Then $L(\chi, s g n)$ equals the standard module representation $X\left(\chi, e_{\max }, \phi=\right.$ triv $)$. In particular, if the orbit of e meets a Levi coponent of a proper parabolic subalgebra, then $L(\chi, \operatorname{sgn})$ is induced irreducible from the corresponding $L_{M}(\chi, \mathrm{sgn})$.

Modulo the characters of tempered representations (for which there is a formula in [6]) this theorem computes the character of $L(\chi, \mathrm{sgn})$. Applying $\mathcal{I} \mathcal{M}$, we get a corresponding result for spherical representations.

\subsection{UNITARY REPRESENTATIONS}

One of the main features of Theorem 1.1 is that it implies that $\mathcal{I M}$ preserves unitarity. A special case of the representations $X(\chi, \operatorname{sgn})$ is when in fact $(h, e)$ can be made into a Lie triple, i.e., there is $f$ such that $[h, e]=2 e,[h, f]=$ $-2 f,[e, f]=h$. Then $X(\chi, \operatorname{sgn})$ consists of the $\mathcal{I}$-fixed vectors of a tempered representation, therefore unitary. These are special cases of Arthur parameters. The Main Theorem says that they are essentially the only unitary representations containing sgn.

\subsection{SIGNATURE AT INFINITY}

This is a technique first used by Casselman [4]. We recast it in terms of graded Hecke algebras and sharpen it.

Recall that the signature of a Hermitian module $L$ is the formal sum

$$
\operatorname{Sign}[L]:=\sum_{\mu \in \widehat{W}}\left([L: \mu]_{+}-[L: \mu]_{-}\right) \mu
$$

Suppose we are in the situation of 1.8, in particular the cases in Examples 1 and 2. We will assume that $\mathcal{V}=\mathcal{W} \otimes \mathbb{H}_{\nu}$ satisfies (1.5.4) with $\nu$ real and $L(M, \mathcal{W}, \nu)$ admits a nondegenerate Hermitian form, but not necessarily unitary. 
Consider the module $I(M, \mathcal{W}, \nu)$ as a $\mathbb{C}[W]$ module only. This module admits another $(\mathbb{C} W$ invariant only) Hermitian form

$$
\left\{t_{x} \otimes v_{x}, t_{y} \otimes v_{y}\right\}:=\left\langle\varepsilon\left(t_{y^{-1}} t_{x} t_{m}\right) \tau\left(v_{x}\right), v_{y}\right\rangle_{K} .
$$

As before, this has a signature.

LEMMA. Suppose $\nu \gg 0$. Then the signature of $\langle$,$\rangle coincides with the signature$ of $\{$,$\} .$

Proof. Suppose that $\nu$ is one-dimensional to simplify the notation. Multiply the formula in Corollary 1.8 by $\nu^{-\left|\Delta^{+}\right|+\left|\Delta^{+}(M)\right|}$ and let $\nu \rightarrow \infty$. Then $r_{w}$ tends to $t_{m}$. Since the coefficients in the signature are integers, they do not change in the limit and so the claim follows.

\subsection{A NONUNITARY CRITERION}

For the above formula to be useful, we need to compute $\{$,$\} on isotypic com-$ ponents. We place ourselves in the cases of Examples 1 and 2. Fix an irreducible $W(M)$ module $\left(\mu_{M}, V_{M}\right)$ and a positive definite invariant form $\langle,\rangle_{V_{M}}$ (with respect to $W(M)$ only). In types $B_{m}, C_{m}$, let $\tau_{M}=$ Id: $V_{M} \rightarrow V_{M}$. In type $D_{m}$, suppose $\mu_{M}$ is invariant under the outer automorphism of order 2 . Then $\mu_{M}$ extends to an irreducible representation of $W\left(B_{m}\right)$. The outer automorphism is realized by the action of the short simple root reflection; denote the corresponding automorphism on $V_{M}$ by $\tau_{M}$.

Let

$$
I\left(W(M), \mu_{M}\right):=\operatorname{Ind}_{W(M)}^{W}\left[V_{M}\right] .
$$

This inherits a form

$$
\left(t_{x} \otimes v_{x}, t_{y} \otimes v_{y}\right):=\left\langle\varepsilon\left(t_{y^{-1}} t_{x}\right) \tau_{M}\left(v_{x}\right), v_{y}\right\rangle_{V_{M}} .
$$

We are interested in the signature of this form.

Given an irreducible representation $(\mu, V)$ of $W$ (typically a constituent of $I\left(W(M), \mu_{M}\right)$ ), we fix a positive $W$-invariant Hermitian form as well. Denote by $d(\mu)$ the lowest degree so that $\mu$ occurs in the representation on harmonic polynomials on $S(\mathfrak{t})$.

PROPOSITION. Suppose $M=\mathrm{GL}(k) \times G(m)$ and $\mu_{M}=\operatorname{sgn} \otimes V_{M}$. Then $I\left(W(M), \mu_{M}\right)$ decomposes with multiplicity 1 . Suppose furthermore that $\mu_{M}$ is invariant under the automorphism of order two in Example 2 of 1.8 in type $D_{m}$, and similarly $\mu$ is invariant under the corresponding automorphism of order two in type $D_{n}$. The form (2.4.2) satisfies

$$
\operatorname{Sign}\left[I\left(W(M), \mu_{M}\right): \mu\right]= \pm(-1)^{d(\mu)+d_{G(m)}\left(\mu_{M}\right)} .
$$

The sign only depends on $W$ and $W(M)$. 
Proof. Assume that we are in the case B or C. Then $\tau_{M}=\mathrm{Id}$ and $t_{0}=t_{m} t_{0}^{M}$ as well as $t_{0}^{M}$ act by scalars on $V$ and $V_{M}$ respectively. The signature is the trace of the action of $t_{m}$ on the $\mu$-isotypic component of $I\left(W(M), \mu_{M}\right)$. This turns out to be the product of the aforementioned scalars. These scalars can be computed using the explicit realizations of the modules as in [7]. Up to a scalar equal to \pm 1 depending on $m, n$ only, it is the claimed formula.

Type $D_{n}$ reduces to type $B_{n}$. Embed $W\left(D_{n}\right)$ in $W\left(B_{n}\right)$ in the usual way. Extend the modules $\mu_{M}$ and $\mu$ to irreducible modules for $W\left(B_{n}\right)$. The long element in $W\left(D_{n}\right)$ is in the center only when $n$ is even. A case by case analysis of the possible parities of $n, m$ and the formula for $\tau_{M}$, shows that the signature (2.4.2) comes down to computing the trace of $t_{m}$ for type B.

COROLLARY 1. Suppose $\mu, \mu^{\prime}$ and $\mu_{M}$ are as in the proposition, i.e.,

$-\mu_{M}$ is a representation of $W(M)$,

$-\mu, \mu^{\prime}$ are representations of $W$ occurring in $I\left(W(M), \mu_{M}\right)$. Assume they occur with multiplicity 1 in $I(M, \mathcal{W}, \nu)$ and $\mu_{M}$ satisfies

$$
1=\left[\mu_{M}: \mathcal{W}\right]=\left[\left.\mu\right|_{W(M)}: \mu_{M}\right]=\left[\left.\mu^{\prime}\right|_{W(M)}: \mu_{M}\right] .
$$

If $\mu$ is written $\left(x_{0}, \ldots\right) \times\left(x_{1}, \ldots\right)$ and $\mu^{\prime}$ is written as $\left(x_{0}^{\prime}, \ldots\right) \times\left(x_{1}^{\prime}, \ldots\right)$, then the signature (2.3.2) on $V$ differs from the signature on $V^{\prime}$ by the factor

$$
(-1)^{\sum x_{2 i+1}} \cdot(-1)^{\sum x_{2 i+1}^{\prime}}
$$

Proof. Since $\mu_{M}$ occurs with multiplicity 1, $\tau$ in formula (2.3.2) differs from $\tau_{M}$ differ by a scalar multiple, so we can use (2.4.2). The formula is a consequence of the explicit formulas for the lowest harmonic degree.

The next corollary is the crucial ingredient in determining the unitary dual of type A. Since as already mentioned this case was done in [10], we omit all details.

COROLLARY 2. Suppose $M=\mathrm{Gl}(k) \times \mathrm{Gl}(k)$ and $\mu_{M}=\operatorname{sgn} \otimes \operatorname{sgn}$. Then $I\left(W(M), \mu_{M}\right)$ decomposes with multiplicity 1 and

$$
\operatorname{Sign}_{K}\left[I\left(W(M), \mu_{M}\right): \mu\right]=(-1)^{d(\mu)} .
$$

This is up to a sign which is independent of the choice of $\mu$ or $\mu_{M}$.

Proof. Omitted.

\section{Description of the Spherical Parameters}

\subsection{EXPLICIT LANGLANDS PARAMETERS}

We consider the spherical dual of the split classical groups of rank $n$ of type B, C, D, precisely $G=\operatorname{So}(2 n+1), G=\operatorname{Sp}(2 n)$ and $G=\operatorname{So}(2 n)$ and their spherical 
unitary duals. We parametrize everything in terms of the dual group, e.g., Type B means that the p-adic group is $S o(2 n+1)$, but the parametrization is in terms of $\mathrm{Sp}(2 n)$. Furthermore, we work in the equivalent setting of representations which contain the sgn K-type rather than triv, and everything is in terms of the graded Hecke algebra. Representations of the Weyl group are represented as pairs of partitions with other conventions being as in [7]. The infinitesimal character is assumed real.

We now give explicit descriptions of the Langlands parameters of the $L(\chi, \operatorname{sgn})$. We do not need to consider all possible nilpotent orbits, but rather a subset which contains all the cases where $\mathcal{W}$ is not unitarily induced irreducible from a similar parameter on a proper Levi component. In general, the Levi component of the parameter of $L(\chi, \mathrm{sgn})$ is of the form $\prod \mathrm{Gl}\left(n_{i}\right) \times G(m)$ where $G(m)$ is a group of classical type other than $\mathrm{Gl}(n)$. The representation on a $\mathrm{Gl}\left(n_{i}\right)$ factor is the Steinberg representation tensored with a character. The representation on the $G(m)$ factor is tempered irreducible containing sgn. To describe the parameters on the $\mathrm{Gl}\left(n_{i}\right)$ factors we introduce the following notation which is a variant of the one used by Zelevinski.

DEFINITION. A string is a sequence of numbers $(a, a+1, \ldots, b-1, b)$ with numbers increasing by 1 from $a$ to $b$. A set of strings is called nested if the entries of any two such strings $\left(a_{1}, \ldots, b_{1}\right)$ and $\left(a_{2}, \ldots, b_{2}\right)$ differ by integers and either

$$
a_{1} \leqslant a_{2} \leqslant b_{2} \leqslant b_{1} \quad \text { or } \quad a_{2} \leqslant a_{1} \leqslant b_{1} \leqslant b_{2},
$$

or else

$$
b_{1}+1<a_{2} \text { or } b_{2}+1<a_{1} .
$$

Each string represents the Steinberg representation tensored with a character on a $\mathrm{Gl}\left(n_{i}\right)$ with $n_{i}=b_{i}-a_{i}+1$. A set of strings represents an induced module from a representation $\otimes\left[S t \otimes \chi_{i}\right]$ on Levi component $\prod \mathrm{Gl}\left(n_{i}\right)$. If the strings are nested, there is no way of combining the entries of any two such strings to form a strictly longer one. This property has to do with Theorem 2.1; the induced module is irreducible and of course contains sgn. On the other hand, take a set of entries, and interpret it as an infinitesimal character $\chi$ of some $\mathrm{Gl}(n)$. Then there is only one way to make a nested set of strings. The ensuing

$$
\left(\prod \mathrm{Gl}\left(n_{i}\right), \mathrm{St} \otimes \chi_{i}\right)
$$

is the Langlands parameter of the $L(\chi, \mathrm{sgn})$ in Theorem 2.1.

The tempered parameter on $G(m)$ is listed in the course of Section 3.2. It is in terms of the partition attached to the nilpotent orbit figuring in the KazhdanLusztig parametrization. We do the special case when the infinitesimal character 
has the same integrality as the tempered one on $G(m)$ in Section 3.2. In 3.3, we deal with the most general case.

\subsection{A SPECIAL CASE}

We consider the case when the infinitesimal character has the same integrality as the trivial representation. This means that if the Kazhdan-Lusztig parameter is $(s, \mathcal{O})$ (Section 1.2), then $s$ has no elliptic part and the hyperbolic part differs from the infinitesimal character of the trivial representation (which we normalize to be ${ }^{L} \rho$ ) by an element in the root lattice of ${ }^{L} G$. This kind of infinitesimal character contains the most interesting tempered representations.

We now describe a typical parameter for $L(\chi, \mathrm{sgn})$. The first part of the data consists of a nilpotent orbit $\mathcal{O}$ in $\mathfrak{g}(m)$ which is even. Attached to this there is a unique tempered irreducible representation which contains the sgn K-type. The semisimple element of the corresponding Lie triple gives its infinitesimal character. The other part is given by a collection of strings. Conditions (3.2.5), (3.2.10) and (3.2.15) on these strings are the analogues of being nested in the case of $\mathrm{Gl}(n)$. The nilpotent orbit for the full Langlands parameter is obtained by adding a pair $\left(n_{i}, n_{i}\right)$ for each string in (3.2.5), (3.2.10) and (3.2.15) to the partition of the nilpotent coming from the parameter on $G(\mathrm{~m})$. If this condition is not satisfied, then it is possible to find a Langlands parameter for a strictly larger nilpotent orbit at the given infinitesimal character.

Remark. The $S$-symbol refers to the algorithms in [9] Sections 11-13. It is used in determining the Weyl group representation attached to a given character of the component group of a nilpotent orbit by the Springer correspondence. The algorithms also give the lowest K-types of the standard modules attached to $\mathcal{O}$. However it should be noted that the K-types of the standard module $X$ corresponding to the nilpotent orbit $\mathcal{O}$ are $H^{*}\left(\mathcal{B}_{\mathcal{O}}\right) \otimes$ sgn. This accounts for the $\otimes$ sgn appearing in all the formulas.

Type B. Consider the nilpotent orbit with partition

$$
\mathcal{O} \leftrightarrow\left(2 x_{0}, 2 x_{1}, \ldots, 2 x_{2 m}\right), \quad 0 \leqslant x_{0} \leqslant \cdots \leqslant x_{i} \leqslant x_{i+1} \leqslant \cdots .
$$

It gives rise to a (unique) irreducible Tempered Representation with lowest K-type

$$
\mu_{\mathcal{O}}=\left(x_{0}, x_{2}, \ldots, x_{2 m}\right) \times\left(x_{1}, x_{3}, \ldots, x_{2 m-1}\right) \otimes \operatorname{sgn}
$$

corresponding to the trivial character of the component group of the orbit $\mathcal{O}$. The $S$-symbol is

$$
\left(\begin{array}{l}
x_{0} \\
x_{1}+1^{x_{2}+2} x_{3}+3^{\cdots} \cdots x_{2 m-1}+2 m-1
\end{array}\right.
$$

The infinitesimal character of the parameter is

$$
\left(1 / 2, \ldots, x_{0}-1 / 2,1 / 2, \ldots, x_{1}-1 / 2, \ldots, 1 / 2, \ldots, x_{2 m}-1 / 2\right)
$$

(where the first string does not appear if $x_{0}=0$ ). 
The remainder of the parameter is given by several strings of the form $\left(e_{i}^{j}-1 / 2, \ldots, E_{i}^{j}-1 / 2\right), \quad x_{j}<e_{i}^{j}-1<E_{i}^{j}<x_{j+1}+1,\left|e_{i}^{j}\right| \leqslant\left|E_{i}^{j}\right|$ satisfying

(1) the representation is induced irreducible from a representation on the Levi component of a $P=\Pi \mathrm{Gl}(*) \times \mathrm{So}(*)$ which is a tensor product of Steinberg Representations on the Gl-factors (modified by a character on the center to represent a string as above) with an irreducible Tempered Representation on the So-factor,

(2) the strings are nested and the Tempered Representation on the So-factor contains the sgn K-type. Each string as in (3.2.5) contributes a pair $\left(E_{i}^{j}-\right.$ $\left.e_{i}^{j}+1, E_{i}^{j}-e_{i}^{j}+1\right)$ to the partition of the nilpotent orbit parametrizing the representation.

Condition (1) is just an explicit description of the Langlands parameter. Condition (2) is an explicit description of the assumptions and conclusions of Theorem 2.1. In (3.2.5), we are using the convention that $x_{j}=\infty$ for $j>2 \mathrm{~m}$.

Type $C$. Consider the nilpotent orbit with partition

$$
\mathcal{O} \leftrightarrow\left(2 x_{0}+1,2 x_{1}+1, \ldots, 2 x_{2 m}+1\right), \quad x_{i} \leqslant x_{i+\mathbf{i}} .
$$

It gives rise to a (unique) irreducible Tempered Representation with lowest K-type

$$
\mu_{\mathcal{O}}=\left(x_{0}, x_{2}, \ldots, x_{2 m}\right) \times\left(x_{1}+1, x_{3}+1, \ldots, x_{2 m-1}+1\right) \otimes \operatorname{sgn}
$$

corresponding to the trivial character of the component group of $\mathcal{O}$. The $S$-symbol is

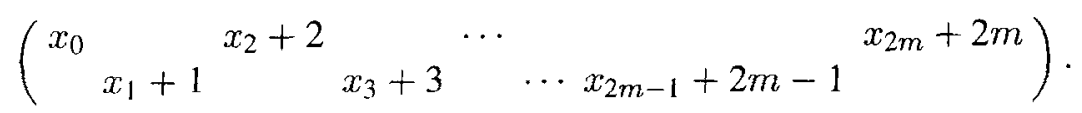

The infinitesimal character of the parameter is

$$
\left(1, \ldots, x_{0}, \quad 0,1, \ldots, x_{1}, \ldots, 0,1, \ldots, x_{2 m-1}, \quad 1, \ldots, x_{2 m}\right)
$$

(if $x_{0}=0$, the first string doesn't appear).

The remainder of the parameter is given by several strings of the form

$\left(e_{i}^{j}, e_{i}^{j}+1, \ldots, E_{i}^{j}\right), \quad x_{j}<e_{i}^{j}-1<E_{i}^{j}<x_{j+1}+1,\left|e_{i}^{j}\right| \leqslant\left|E_{i}^{j}\right|$

satisfying

(1) the representation is induced irreducible from a representation on the Levi component of a $P=\prod \mathrm{Gl}(*) \times \mathrm{Sp}(*)$ which is a tensor product of Steinberg Representations on the Gl-factors (modified by a character on the center to represent a string as above) with an irreducible Tempered Representation on the $S p$-factor, 
(2) the strings are nested and the representation on the Sp-factor contains the sgn $\mathrm{K}$-type. Each string as in (3.2.10) contributes a pair $\left(E_{i}^{j}-e_{i}^{j}+1, E_{i}^{j}-e_{i}^{j}+1\right)$ to the partition of the nilpotent orbit parametrizing the representation.

Condition (1) is an explicit description of the Langlands parameter. Condition (2) is an explicit description of the assumptions and conclusion of Theorem 2.1. In (3.2.10), we are using the convention that $x_{j}=\infty$ for $j>2 \mathrm{~m}$.

Type D. Consider the nilpotent orbit with partition

$$
\mathcal{O} \leftrightarrow\left(2 x_{0}+1,2 x_{1}+1, \ldots, 2 x_{2 m-1}+1\right), \quad x_{i} \leqslant x_{i+1} .
$$

It gives rise to a (unique) irreducible Tempered Representation with lowest K-type

$$
\begin{aligned}
\mu_{\mathcal{O}}= & \left(x_{0}, x_{2}, \ldots, x_{2 m-2}\right) \times \\
& \times\left(x_{1}+1, x_{3}+1, \ldots, x_{2 m-1}+1\right) \otimes \operatorname{sgn}
\end{aligned}
$$

corresponding to the trivial character of the component group of $\mathcal{O}$. The $S$-symbol is

$$
\left(\begin{array}{cccc}
x_{0} & x_{2}+2 & \cdots & x_{2 m-2}+2 m-2 \\
x_{1}+1 & x_{3}+3 & \cdots & x_{2 m-1}+2 m-1
\end{array}\right) .
$$

The infinitesimal character of the parameter is

$$
\left(0,1, \ldots, x_{0}, 1, \ldots, x_{1}, \ldots, 1, \ldots, x_{2 m-1}\right) .
$$

The remainder of the parameter is given by several strings of the form

$$
\left(e_{i}^{j}, \ldots, E_{i}^{j}\right), \quad x_{j}<e_{i}^{j}-1<E_{i}^{j}<x_{j+1}+1,\left|e_{i}^{j}\right| \leqslant\left|E_{i}^{j}\right|
$$

satisfying

(1) the representation is induced irreducible from a representation on the Levi component of a $P=\prod \mathrm{Gl}(*) \times \mathrm{So}(*)$ which is a tensor product of Steinberg Representations on the Gl-factors (modified by a character on the center to represent a string as above) with an irreducible Tempered Representation on the So-factor,

(2) the strings are nested and the Tempered Representation on the So-factor contains the sgn K-type. Each string as in (3.2.15) contributes a pair $\left(E_{i}^{j}-\right.$ $\left.e_{i}^{j}+1, E_{i}^{j}-e_{i}^{j}+1\right)$ to the partition of the nilpotent orbit parametrizing the representation.

Condition (1) is an explicit description of the Langlands parameters. Condition (2) is an explicit description of the assumptions and conclusions of Theorem 2.1. In (3.2.15), we are using the convention that $x_{j}=\infty$ for $j>2 \mathrm{~m}$. 


\subsection{THE GENERAL CASE}

In addition to the tempered part there are also strings $(f+\nu, \ldots, F+\nu)$ whose coordinates do not have the same integrality as in (3.2). To deal with them systematically, we make the following definition.

DEFINITION. We say a string $(f+\nu, \ldots, F+\nu)$ is of type $\varepsilon$ if it is

- of even length in type $B$,

- of odd length in type C, D.

In the first case we set $\varepsilon=1 / 2$, while in the second case we set $\varepsilon=0$.

Then we can write all these remaining strings in the form

$$
\left(f_{j}+\nu_{j}, \ldots, F_{j}+\nu_{j}\right), \quad f_{j}, F_{j} \equiv \varepsilon(\mathbb{Z}), 0<\nu_{j} \leqslant 1 / 2 .
$$

For a given $\nu_{j}$, the strings are nested and the representation is induced irreducible from a Levi component of a parabolic subalgebra as in the case of integer strings. This representation of the strings is unique except when $\nu_{j}=1 / 2$; here is the reasoning. Observe that any string can be written as

$$
(f+\nu, \ldots, F+\nu)
$$

with $f, F \equiv \varepsilon(\mathbb{Z})$ and $0<\nu<1$. If $\nu>1 / 2$, then replace it by $1-\tilde{\nu}$ and reverse the signs and order to get

$$
(-F-1+\tilde{\nu}, \ldots,-f-1+\tilde{\nu})
$$

which is of the form (3.3.1). In case $\nu_{j}=1 / 2$, we can represent each string as either $(f+1 / 2, \ldots, F+1 / 2)$ or $(-F-1+1 / 2, \ldots,-f-1+1 / 2)$. We choose the expression with the absolute value of the leftmost number being larger, i.e., (3.3.2) if $|F| \leqslant|f|$, and (3.3.3) if $|f+1| \leqslant|F+1|$. We apply the same conventions to the strings of the form (3.2.5), (3.2.10) and (3.2.15). The restrictions on the $x$ 's and the $e$ 's can be written uniformly as

$$
x_{j}+1+\varepsilon<e_{i}<E_{i}<x_{j+1}+1-\varepsilon .
$$

Remarks. (1) We may and do incorporate the strings of integers as in (3.2.5), (3.2.10) and (3.2.15) into the strings of the type as above, by applying a small deformation. In other words, the ${ }^{L} \rho$-like part of the parameter is always assumed tempered.

(2) In type D, a (real) spherical parameter $\lambda$ for So is Hermitian if and only if there is $w \in W\left(D_{n}\right)$ such that $w \lambda=-\lambda$. If the parameter has entries equal to zero, then the analysis above is sufficient. If not, there are two inequivalent spherical parameters, one for $\lambda$ and another for $\lambda^{\prime}$ obtained by applying the outer 
automorphism of order two. They are either both unitary or both nonunitary. In the description above, one string might have to be written as

$$
(-f-\nu, f+1+\nu, \ldots, F+\nu) \text {. }
$$

If so, we can always consider the other parameter.

\subsection{RELATION BETWEEN INFINITESIMAL CHARACTERS AND STRINGS}

We also need to show how to obtain this parametrization in terms of strings from the infinitesimal character (cf. Theorem 2.1).

Type $B$. Write the half-integer part in increasing order

$$
\begin{aligned}
& (r-1 / 2, \ldots, r-1 / 2, \ldots, R-1 / 2, \ldots, R-1 / 2), \\
& \quad 0<r \leqslant R \text { integers. }
\end{aligned}
$$

Then extract the longest possible string $(r-1 / 2, \ldots, l-1 / 2)$. The remaining parameter is of the same form, so we can continue. The strings that start with $1 / 2$ form the tempered part of the parameter.

For the integer part, write it in increasing order as

$$
(r, \ldots, r, \ldots, R, \ldots, R), \quad 0 \leqslant r \leqslant R \text { integers. }
$$

Extract the largest possible string $(l, \ldots, 0, \ldots, k)$ (or just $(l, \ldots, k)$ with $l>0$ if there are no entries equal to 0 ) by changing entries to their negatives if necessary. Assume as we may that $k \geqslant|l|$. The remainder is of the same form as in (3.4.2), so we can continue until there are no entries left. Then rewrite the strings in increasing order as in 3.3 .

For the other parts, group them according to distinct $\nu$ 's as

$$
\begin{gathered}
(r+\nu, \ldots, r+\nu, \ldots, R+\nu, \ldots, R+\nu), \\
\text { with } 0<\nu<1 / 2, r \leqslant R \text { integers. }
\end{gathered}
$$

Then extract the longest possible string $(r+\nu, \ldots, l+\nu)$ and rewrite it as in 3.3. The remainder is of the same type, so extract strings in the same way until there are no entries left.

Type $C$. Write the integer part in increasing order

$$
(r, \ldots, r, \ldots, R, \ldots, R), \quad 0 \leqslant r \leqslant R .
$$

Extract the longest possible string $(l, \ldots, 0, \ldots, k)$ with the same conventions as for type B right after (3.4.2). The remaining parameter is of the same form, so we can continue until there are no zeroes left. The number of strings is the $m$ for the tempered part of the parameter and, furthermore, $k=x_{2 m}, l=x_{2 m-1}$. After that, extract the longest possible strings of the form $(l, \ldots, k)$ with $k \geqslant l>0$ 
until there are no entries left. If the longest such string ends in a 1 , then its largest entry is $x_{0}$, otherwise set $x_{0}=0$.

For the half-integer part, write it as

$$
(r-1 / 2, \ldots, r-1 / 2, \ldots, R-1 / 2, \ldots, R-1 / 2), 0 \leqslant r \leqslant R .
$$

Then extract the longest possible string of the form $(l-1 / 2, \ldots, k+1 / 2)$, by changing entries into their negatives if necessary. The remainder is of the same form as in (3.4.5), so we can continue until there are no entries left. Rewrite the strings as in 3.3 .

For the rest of the parameter which is formed of neither integers nor halfintegers, group them according to distinct $\nu$ 's as

$$
\begin{gathered}
(r+\nu, \ldots, r+\nu, \ldots, R+\nu, \ldots, R+\nu) \\
\text { with } 0<\nu<1 / 2, \quad r \leqslant R \text { integers. }
\end{gathered}
$$

Then extract the longest possible string $(r+\nu, \ldots, l+\nu)$. The remainder is of the same type, so extract strings until there are no entries left.

Type $D$. Write the integer part in increasing order

$$
(r, \ldots, r, \ldots, R, \ldots, R), \quad 0 \leqslant r \leqslant R .
$$

Extract the longest possible string $(l, \ldots, 0, \ldots, k)$ with the same conventions as for type B right after (3.4.2). The remaining parameter is of the same form, so we can continue until there are no zeroes left. The number of strings is the $m$ for the tempered part of the parameter. Furthermore, assuming as we may that $|l| \leqslant k$, we get $k=x_{2 m}, l=x_{2 m-1}$. After that, extract the longest possible strings of the form $(l, \ldots, k)$ with $k \geqslant l>0$ until there are no entries left.

For the half-integer part, write it as

$$
(r-1 / 2, \ldots, r-1 / 2, \ldots, R-1 / 2, \ldots, R-1 / 2), \quad 0 \leqslant r \leqslant R .
$$

Then extract the longest possible string of the form $(l-1 / 2, \ldots, k+1 / 2)$, by changing entries into their negatives if necessary. The remainder is of the same form as in (3.4.8), so we can continue until there are no entries left. Rewrite the strings as in 3.3 .

For the rest of the parameter which is formed of neither integers nor halfintegers, group them according to distinct $\nu$ 's as

$$
\begin{gathered}
(r+\nu, \ldots, r+\nu, \ldots, R+\nu, \ldots, R+\nu), \\
\text { with } 0<\nu<1 / 2, m \leqslant M \text { integers. }
\end{gathered}
$$

Then extract the longest possible string $(r+\nu, \ldots, l+\nu)$. The remainder is of the same type, so extract strings until there are no entries left.

Note also that Remark (2) from 3.3 applies. 


\section{The Main Result}

\subsection{PARAMETERS OF UNITARY REPRESENTATIONS}

The main result is easy to state, but the parameters are hard to describe explicitly.

THEOREM. A parameter as above is unitary if and only if it is a complementary series from an induced from a tempered representation tensored with a Gl-complementary series.

We will prove this theorem by induction, increasing in the rank of the group, decreasing in the dimension of the nilpotent orbit parametrizing the representation.

We now describe these parameters in precise terms. We assume the conventions in Section 3. There are several conditions, (A), (B) and (C1)-(C4).

(A) The ${ }^{L} \rho$-like part of the parameter is tempered.

(B) Any string not of type $\varepsilon$ is of the form

$$
(-E-1+\nu, \ldots, E+\nu), \quad 0<\nu \leqslant 1 / 2, E \equiv \varepsilon(\mathbb{Z}) .
$$

Any string of type $\varepsilon$ is of the form

$$
(-E+\nu, \ldots, E+\nu)
$$

or

$$
(-E-1+\nu, \ldots, E-1+\nu), \quad 0<\nu \leqslant 1 / 2, E \equiv \varepsilon(\mathbb{Z}) .
$$

We will want to deform the $\nu$ 's in various intervals so that the representation stays induced irreducible, but we will also consider the endpoint of such an interval and the factor containing sgn. In particular, deforming $\nu$ in (4.1.1a) to $1 / 2$ gives a unitarily induced irreducible representation. But deforming $\nu$ in (4.1.1b) to 0 or $\nu$ in (4.1.1c) to 1 does not necessarily give an induced irreducible representation (cf. (C4)).

DEFINITION. Consider two strings as in (4.1.1) with parameters $\nu_{1} \leqslant \nu_{2}$. We say that they are adjacent, if they have the same $E$ and $\nu_{1}=\nu_{2}$, or $\nu_{1}<\nu_{2}$ and there is no other string with the same $E$ and parameter $\nu_{1} \leqslant \nu \leqslant \nu_{2}$.

LEMMA. In $\mathrm{Gl}(n)$, the representation corresponding to the pair of strings ( $-E-$ $\nu, \ldots, E-\nu)$ and $(-E+\nu, \ldots, E+\nu)$ is a complementary series for $\nu<\frac{1}{2}$, and is not unitary for $\nu>\frac{1}{2}, \nu \notin \frac{1}{2} \mathbb{Z}$.

Proof. This is well known from [10]; it also follows from Corollary 1 of 2.4.

Assume that (A) and (B) are satisfied. Consider the string (4.1.1a) with $0<$ $\nu<1 / 2$ and deform $\nu$ upwards to $1 / 2$. We claim that no reducibility can occur. 
Indeed, if it did, there would have to be another string $\left(f+\nu^{\prime}, \ldots, F+\nu^{\prime}\right)$ such that $F+\nu^{\prime}-E-\nu^{\prime \prime}$ or $F+\nu^{\prime}+E+\nu^{\prime \prime}$ is an integer and

$$
-E-1+\nu^{\prime \prime}<f+\nu^{\prime}, \quad E+\nu^{\prime \prime}<F+\nu^{\prime},
$$

or

$$
f+\nu^{\prime}<-E-1+\nu^{\prime \prime}, \quad F+\nu^{\prime}<E+\nu^{\prime \prime}
$$

for some $\nu \leqslant \nu^{\prime \prime}<1 / 2$. Neither case can happen. Because of (3.3.1), $E, F \cong$ $\varepsilon(\mathbb{Z})$, and $0<\nu^{\prime} \leqslant 1 / 2$. It follows that $\nu^{\prime}-\nu^{\prime \prime}$ or $\nu^{\prime}+\nu^{\prime \prime} \in \mathbb{Z}$, in fact it must be the case that $\nu^{\prime}=\nu^{\prime \prime}$. Since $\left(f+\nu^{\prime}, \ldots, F+\nu^{\prime}\right)$ is assumed to satisfy (4.1.1), we find that (4.1.2) cannot hold.

Thus, we can remove strings of type (4.1.1a) by a complementary series argument.

Consider the strings of type (4.1.1b). If there are two adjacent strings of this type, then by Lemma 4.1 the representation is a complementary series from a representation which is unitarily induced from a Levi component of type $\mathrm{Gl}(4 E+$ 2) $G(*)$ and a Gl-complementary series.

We can summarize these two properties in the following.

(C1) Any string not of type $\varepsilon$ is in a complementary series from an induced from Triv $\otimes *$ on a $\mathrm{Gl}(2 E+2) G(*)$. Any two adjacent strings of type $\varepsilon$ as in (4.1.1b) are in a complementary series from a $\mathrm{Gl}(4 E+2)$.

These strings can be removed from the parameter; the ensuing parameter is unitary if and only if the original parameter is unitary.

A similar reasoning applies for strings of type (4.1.1c). The nonunitarity part of Lemma 4.1 gives the following.

(C2) If two strings of type (4.1.1c) are adjacent, the parameter is not unitary.

Thus, consider the case when (A), (B), (C1) and (C2) hold. By removing strings we are reduced to the case when for a given $E$, there is at most one string for each value of $\nu$; and they must alternate between (4.1.1b) and (4.1.1c) with increasing $\nu$. Suppose there is more than one string present, and label the $\nu$ 's as $0<\nu_{1}<\nu_{2}<\cdots<\nu_{m-1}<\nu_{m}$. Suppose the one with parameter $\nu_{m}$ is of type (4.1.1c). Then we can deform $\nu_{m}$ upwards to $1-\nu_{m-1}$, and see that this is induced irreducible from a representation which is unitarily induced from a Levi component $\mathrm{Gl}(4 E+2) G(*)$ (a complementary series on $\mathrm{Gl}(4 E+2)$ ). Such a pair can be removed from the parameter; the ensuing parameter is unitary if and only if the original parameter is unitary.

On the other hand, suppose the string with parameter $\nu_{m}$ is of type (4.1.1b). Deforming $\nu_{m}$ upwards to $1-\nu_{m-1}$, gives a parameter that is not unitary by (C2). Thus, 
(C3) Suppose there is more than one string (for some given $E$ ). If the last string is of type $(4.1 .1 \mathrm{~b})$, then the parameter is not unitary. If the last string is of type (4.1.1c), then the representation is a complementary series from a representation which is unitarily induced from a parabolic subgroup with Levi component $\mathrm{Gl}(4 E+2 \varepsilon) G(*)$. We can remove such pairs of strings, starting with the largest $\nu_{m}$; the ensuing parameter is unitary if and only if the original parameter is unitary.

We are reduced to the case when there is only one string. Deform its $\nu$ to 0 in case (4.1.1b) and to 1 in case (4.1.1c). Reducibility of tempered parameters gives the following.

(C4) In the case of a single string of type (4.1.1b) or (4.1.1c) of size $E$, there must be at least one $x_{i}=E+\varepsilon$ in the tempered parameter.

Thus to see whether a parameter is unitary, one checks whether $(A)-(C)$ are satisfied. First we check that (A) and (B) are satisfied. If so, then remove the Gl-complementary series in step (C1). Then check for adjacent strings of type (4.1.1c) as in (C2). If none are present, remove the complementary series from step (C3). What should result is either a parameter which is tempered or one as in (C4).

Remark. In the case of type D, the condition for a representation to be Hermitian is different from types $\mathrm{B}$ and $\mathrm{C}$. There is no change to the argument for $(\mathrm{C} 1),(\mathrm{C} 2)$ and $(\mathrm{C} 3)$, because they involve pairs of strings. For (C4), if there is a single string present, then the tempered part of the parameter must be nontrivial, otherwise the parameter is not Hermitian. Thus the argument is unchanged.

\subsection{THE CASE OF A SINGLE STRING}

We analyze the unitarity of a parameter such that the ${ }^{L} \rho$-like part is tempered and there is just one other string present. As already remarked, the tempered part of the parameter in type D must be nontrivial. Then there are zeroes in the coordinates, and we may assume that the string is of the form (3.3.2). The argument is the same as for types B, C.

Before plunging into the argument, recall the remark in 3.2 and the algorithms in [9] (an alternative reference is [5]).

The argument is very simple in nature: given a representation whose parameter is the nilpotent orbit $\mathcal{O}$, we find an orbit $\mathcal{O}^{\prime}$ such that

(1) there is no other orbit $\mathcal{O}^{\prime \prime}$ satisfying $\mathcal{O} \subset \overline{\mathcal{O}^{\prime \prime}} \subset \overline{\mathcal{O}^{\prime}}$.

(2) a factor with a lowest K-type attached to $\mathcal{O}^{\prime}$ occurs at the first reducibility point on the half-line $\nu \geqslant 0$.

(3) The lowest K-type $\mu^{\prime}$ of this other factor occurs with multiplicity 1 in $X(\mathcal{W}, \nu)$ and stays in $L(\mathcal{W}, \nu)$ beyond the first reducibility point.

(4) The lowest K-type $\mu$ of $L(\mathcal{W}, \nu)$ and $\mu^{\prime}$ have opposite signatures at $\infty$. 
There some delicate composition series problems that we have to solve. Often arguments that are really the same, are split into two cases (a) and (b), because of the nature of the algorithms in [9].

We consider the case of type $\mathrm{C}$. Then $\varepsilon=0$ and type $\varepsilon$ means an odd sized string. We write the single string as

$$
\begin{array}{r}
\left(-E+\frac{1}{2}+\nu, \ldots, E-\frac{1}{2}+\nu\right), \quad \nu \geqslant 0, \nu+\frac{1}{2} \notin \mathbb{Z}, \\
E \in \mathbb{Z}(\text { not type } \varepsilon), \\
(-E+\nu, \ldots, E+\nu), \quad \nu>0, \nu \notin \mathbb{Z}, E \in \mathbb{Z} \text { (type } \varepsilon) .
\end{array}
$$

This parametrization is different from 3.3, which is designed to deal with several strings.

The representation containing sgn is of the form $X(\mathcal{W}, \nu)=L(\mathcal{W}, \nu)$. If $\mathcal{W}$ is unitarily induced irreducible, then so is $L(\mathcal{W}, \nu)$. We can do the argument on the parameter being induced. Thus we assume as we may that $\mathcal{W}$ is not induced irreducible from any proper Levi component. This means that we may write the nilpotent orbit $\mathcal{O}$ corresponding to $\mathcal{W}$ as in (3.2.6), and in addition for any value $x$, there are at most two $x_{i}=x$. We will show that $L(\mathcal{W}, \nu)$ is not unitary for

$$
\begin{array}{ll}
\nu>1 / 2 & \text { in case (4.2.1a), } \\
\nu \neq 0 & \text { in case (4.2.1b) when no } x_{i}=E, \\
\nu>1 & \text { in case (4.2.1b) when there is an } x_{i}=E .
\end{array}
$$

We deal with the simplest case first, the second case in (4.2.2). The parameter for $L(\mathcal{W}, \nu)$ has nilpotent orbit

$$
\mathcal{O} \leftrightarrow\left(2 x_{0}+1, \ldots, 2 x_{2 k-1}+1,2 x_{2 k}+1=2 E+1,2 x_{2 k+1}=2 E+1, \ldots\right)
$$

or

$$
\left(2 x_{0}+1, \ldots, 2 x_{2 k}+1,2 x_{2 k+1}+1=2 E+1,2 x_{2 k+2}=2 E+1, \ldots\right) \text {. }
$$

The two $x$ 's equal to $E$ correspond to the string (4.2.1) and no other $x$ 's are equal to $E$. In this case $L(\mathcal{W}, \nu)$ has two lowest $\mathrm{K}$-types which occur in the same factor for $\nu>0$. They are

$$
\begin{aligned}
\mu= & \left(x_{0}, \ldots, x_{2 k}=E, \ldots, x_{2 m}\right) \times \\
& \times\left(x_{1}+1, \ldots, x_{2 k+1}+1=E+1, \ldots, x_{2 m-1}+1\right) \otimes \mathrm{sgn}, \\
\mu^{\prime}= & \left(x_{0}, \ldots, x_{2 k}^{\prime}=E+1, \ldots, x_{2 m}\right) \times \\
& \times\left(x_{1}+1, \ldots, x_{2 k+1}^{\prime}+1=E, \ldots, x_{2 m-1}+1\right) \otimes \mathrm{sgn},
\end{aligned}
$$

or

$$
\begin{aligned}
\mu= & \left(x_{0}, \ldots, x_{2 k+2}=E, \ldots, x_{2 m}\right) \times \\
& \times\left(x_{1}+1, \ldots, x_{2 k+1}+1=E+1, \ldots, x_{2 m-1}+1\right) \otimes \mathrm{sgn},
\end{aligned}
$$




$$
\begin{aligned}
\mu^{\prime}= & \left(x_{0}, \ldots, x_{2 k+2}^{\prime}=E-1, \ldots, x_{2 m}\right) \times \\
& \times\left(x_{1}+1, \ldots, x_{2 k+1}^{\prime}+1=E+2, \ldots, x_{2 m-1}+1\right) \otimes \mathrm{sgn} .
\end{aligned}
$$

At $\infty$, the K-types $\mu$ and $\mu^{\prime}$ have opposite signatures. This signature (up to possibly a sign which only depends on the rank of the group) is given by $(-1)^{\sum x_{2 i+1}}$.

We consider the first case of (4.2.2). The standard module is induced irreducible from $\mathrm{GL}(2 E) \mathrm{Sp}(*)$. The nilpotent orbit corresponding to $X(\mathcal{W}, \nu)$ can be written

$$
\mathcal{O} \leftrightarrow\left(2 x_{0}+1, \ldots, 2 x_{2 k-1}+1,2 x_{2 k}=2 E, 2 x_{2 k+1}=2 E, \ldots\right)
$$

or

$$
\left(2 x_{0}+1, \ldots, 2 x_{2 k}+1,2 x_{2 k+1}=2 E, 2 x_{2 k+2}=2 E, \ldots\right) .
$$

The lowest K-type is

$\mu=\left(\ldots, x_{2 k}=E, \ldots\right) \times\left(\ldots, x_{2 k+1}+1=E, \ldots\right) \otimes \operatorname{sgn}$

or

$$
\left(\ldots, x_{2 k+2}=E-1, \ldots\right) \times\left(\ldots, x_{2 k+1}+1=E+1, \ldots\right) \otimes \operatorname{sgn} .
$$

The first reducibility point in this case is at $\nu=1 / 2$. There are (at least) two factors, one corresponding to the nilpotent orbits $\mathcal{O}$, the other to

$$
\begin{aligned}
\mathcal{O}^{\prime} \leftrightarrow\left(\ldots, 2 x_{2 k}+1\right. & =2 E-1, \\
2 x_{2 k+1}+1 & =2 E+1, \ldots) \text { in case }(4.2 .5 \mathrm{a}), \\
\mathcal{O}^{\prime} \leftrightarrow\left(\ldots, 2 x_{2 k+1}\right. & =2 E-1, \\
2 x_{2 k+2}+1 & =2 E+1, \ldots) \text { in case }(4.2 .5 \mathrm{~b}) .
\end{aligned}
$$

The factor corresponding to $\mathcal{O}$ is the lowest $\mathrm{K}$-type factor, the factor corresponding to $\mathcal{O}^{\prime}$ is the factor containing sgn. The lowest K-type for the factor corresponding to $\mathcal{O}^{\prime}$ is

$\mu^{\prime}=\left(x_{0}, \ldots, x_{2 k}^{\prime}=E-1, \ldots\right) \times\left(x_{1}+1, \ldots, x_{2 k+1}^{\prime}+1=E+1, \ldots\right)$

or

$$
\left(x_{0}, \ldots, x_{2 k+2}^{\prime}=E, \ldots\right) \times\left(x_{1}+1, \ldots, x_{2 k+1}^{\prime}+1=E, \ldots\right) .
$$

The two K-types have opposite signature at $\infty$. We need to prove that the corresponding two lowest $\mathrm{K}$-types always stay in the same factor on the half-line $1 / 2<\nu$. This is the same as for the third case, only easier. We omit the details. 
Consider the third case of (4.2.2). Then the nilpotent orbit $\mathcal{O}$ corresponding to $X(\mathcal{W}, \nu)$ has either three or four $x_{i}=E$. In the case of three, we get

$$
\begin{aligned}
\mathcal{O} \leftrightarrow & \left(\ldots, 2 x_{2 k-1}+1=2 E+1,\right. \\
& \left.2 x_{2 k}+1=2 E+1,2 x_{2 k+1}+1=2 E+1, \ldots\right)
\end{aligned}
$$

or

$$
\begin{aligned}
& \left(\ldots, 2 x_{2 k}+1=2 E+1\right. \\
& \left.2 x_{2 k+1}+1=2 E+1,2 x_{2 k+2}+1=2 E+1, \ldots\right) .
\end{aligned}
$$

The lowest $\mathrm{K}$-type is

$$
\begin{aligned}
\mu= & \left(\ldots, x_{2 k}=E, \ldots\right) \times \\
& \times\left(\ldots, x_{2 k-1}+1=E+1, x_{2 k+1}+1=E+1, \ldots\right) \otimes \text { sgn }
\end{aligned}
$$

or

$$
\begin{aligned}
& \left(\ldots, x_{2 k}=E, x_{2 k+2}=E, \ldots\right) \times \\
& \quad \times\left(\ldots, x_{2 k+1}+1=E+1, \ldots\right) \otimes \operatorname{sgn} .
\end{aligned}
$$

The first reducibility point is at $\nu=1$. There are several possible factors, the one we are interested in, corresponds to the nilpotent orbit

$\mathcal{O}^{\prime} \leftrightarrow\left(\ldots, 2 x_{2 k-1}^{\prime}=2 E, 2 x_{2 k}^{\prime}=2 E, 2 x_{2 k+1}^{\prime}+1=2 E+3, \ldots\right)$

or

$$
\left(\ldots, 2 x_{2 k}^{\prime}=2 E, 2 x_{2 k+1}^{\prime}=2 E, 2 x_{2 k+2}^{\prime}+1=2 E+3, \ldots\right) .
$$

The lowest K-type is

$$
\begin{aligned}
\mu^{\prime}= & \left(\ldots, x_{2 k}^{\prime}=E-1, \ldots\right) \times \\
& \times\left(\ldots, x_{2 k-1}^{\prime}+1=E+1, x_{2 k+1}^{\prime}+1=E+2, \ldots\right) \otimes \operatorname{sgn}
\end{aligned}
$$

or

$$
\begin{aligned}
& \left(\ldots, x_{2 k}^{\prime}=E, x_{2 k+2}^{\prime}=E+1, \ldots\right) \times \\
& \times\left(\ldots, x_{2 k+1}^{\prime}=E, \ldots\right) \otimes \operatorname{sgn} .
\end{aligned}
$$

In the case of four $x_{i}=E$, we get

$$
\begin{aligned}
\mathcal{O} \leftrightarrow & \left(\ldots, 2 x_{2 k-1}+1=2 E+1,2 x_{2 k}+1=2 E+1,\right. \\
& \left.2 x_{2 k+1}+1=2 E+1,2 x_{2 k+2}+1=2 E+1, \ldots\right)
\end{aligned}
$$


or

$$
\begin{aligned}
& \left(\ldots, 2 x_{2 k}+1=2 E+1,2 x_{2 k+1}+1=2 E+1,\right. \\
& \left.2 x_{2 k+2}+1=2 E+1,2 x_{2 k+3}+1=2 E+1, \ldots\right) .
\end{aligned}
$$

The lowest K-type of the factor corresponding to $\mathcal{O}$ is

$$
\begin{aligned}
\mu= & \left(\ldots, x_{2 k}=E, x_{2 k+2}=E, \ldots\right) \times \\
& \times\left(\ldots, x_{2 k-1}+1=E+1, x_{2 k+1}=E+1, \ldots\right) \otimes \operatorname{sgn}
\end{aligned}
$$

or

$$
\begin{aligned}
& \left(\ldots, x_{2 k}=E, x_{2 k+2}=E, \ldots\right) \times \\
& \quad \times\left(\ldots, x_{2 k+1}+1=E+1, x_{2 k+3}+1=E+1, \ldots\right) \otimes \operatorname{sgn} .
\end{aligned}
$$

The first reducibility point is at $\nu=1$. There are several possible factors, the one we are interested in corresponding to the nilpotent orbit

$$
\begin{aligned}
\mathcal{O}^{\prime} \leftrightarrow & \left(\ldots, 2 x_{2 k-1}^{\prime}=2 E, 2 x_{2 k}^{\prime}=2 E,\right. \\
& \left.2 x_{2 k+1}^{\prime}=2 E+2,2 x_{2 k+2}^{\prime}=2 E+2, \ldots\right)
\end{aligned}
$$

or

$$
\begin{aligned}
& \left(\ldots, 2 x_{2 k}^{\prime}=2 E, 2 x_{2 k+1}^{\prime}=2 E\right. \\
& \left.2 x_{2 k+2}^{\prime}=2 E+2,2 x_{2 k+3}^{\prime}=2 E+2, \ldots\right) .
\end{aligned}
$$

The lowest $\mathrm{K}$-type is

$$
\begin{aligned}
\mu^{\prime}= & \left(\ldots, x_{2 k}^{\prime}=E-1, x_{2 k+2}^{\prime}=E, \ldots\right) \times \\
& \times\left(\ldots, x_{2 k-1}^{\prime}+1=E+1, x_{2 k+1}^{\prime}+1=E+2, \ldots\right) \otimes \operatorname{sgn}
\end{aligned}
$$

or

$$
\begin{aligned}
& \left(\ldots, x_{2 k}^{\prime}=E, x_{2 k+2}^{\prime}=E+1, \ldots\right) \times \\
& \quad \times\left(\ldots, x_{2 k+1}^{\prime}=E, x_{2 k+3}^{\prime}=E+1, \ldots\right) \otimes \mathrm{sgn} .
\end{aligned}
$$

We claim that, for $\nu>1$, the corresponding lowest K-types always stay in the same factor. At $\infty$ they have opposite signs. In particular, these factors do occur at $\nu=1$. The proof is quite involved.

To compute multiplicities and signatures, we need a restriction formula. 
LEMMA 1. Let $\sigma=\left(b_{0}, \ldots, b_{2 m}\right) \times\left(b_{1}+1, \ldots, b_{2 m-1}+1\right)$ be a K-type. Then the $\mathrm{K}$-types $\sigma^{\prime}$ such that $\operatorname{sgn} \otimes \sigma^{\prime}$ occurs in the restriction of $\operatorname{sgn} \otimes \sigma$ to $\operatorname{Gl}(y) \operatorname{Sp}(*)$ are of the form

$$
\left(b_{0}^{\prime}, \ldots, b_{2 l}^{\prime}, \ldots\right) \times\left(b_{1}^{\prime}+1, \ldots, b_{2 l-1}^{\prime}+1, \ldots\right),
$$

satisfying

$$
b_{2 j-2} \leqslant b_{2 j}^{\prime} \leqslant b_{2 j}, \quad b_{2 j-1} \leqslant b_{2 j-1}^{\prime} \leqslant b_{2 j+1}
$$

and

$$
\sum b_{i}^{\prime}+y=\sum b_{i} .
$$

Furthermore, each such $\sigma^{\prime}$ occurs with multiplicity 1.

Proof. This is a special case of the Littlewood-Richardson rule combined with Mackey theory for semidirect products of groups; we omit the details.

We now reduce to the case when $\mathcal{W}$ is a discrete series, in other words for each value $x$, there is at most one $x_{i}=x$. This fails to be the case whenever there is an $l$ such that $x_{2 l+1}=x_{2 l+2}=x$ or $x_{2 l}=x_{2 l+1}=x$ for some $x$. We consider the case when $x \neq E$. In this case, the lowest K-type of the factor $L(\mathcal{W}, \nu)$ has the form

$$
\left(x_{0}, \ldots, x_{2 l+2}=x, \ldots\right) \times\left(x_{1}+1, \ldots, x_{2 l+1}+1=x+1, \ldots\right)
$$

or

$$
\left(x_{0}, \ldots, x_{2 l}=x, \ldots\right) \times\left(x_{1}+1, \ldots, x_{2 l+1}+1=x+1, \ldots\right) .
$$

As stated before, (4.2.2), no other $x_{i}=x$. Replace $X(\mathcal{W}, \nu)$ by an induced module

$$
X=\operatorname{Ind}_{\mathrm{Sp}(*) \mathrm{Gl}(2 x+1)}\left[X_{1} \otimes \mathrm{St}\right] .
$$

We assume $X_{1}$ is irreducible (containing sgn). The lowest $\mathrm{K}$-type of $X_{1}$ is

$$
\mu_{1}=\left(x_{0}, \ldots, \widehat{x}, \ldots\right) \times\left(x_{1}+1, \ldots, \widehat{x+1}, \ldots\right) .
$$

Then $X$ decomposes into two factors $X_{+}+X_{-}$both corresponding to the same nilpotent orbit (see the remark at the end of 1.2). The first factor is $X(\mathcal{W}, \nu)$ and has lowest $\mathrm{K}$-type given by (4.2.19), the other factor has lowest $\mathrm{K}$-type

$$
\begin{aligned}
& \left(x_{0}, \ldots, x_{2 l+2}=x-1, \ldots\right) \times \\
& \quad \times\left(x_{1}+1, \ldots, x_{2 l+1}+1=x+2, \ldots\right)
\end{aligned}
$$

or

$$
\left(x_{0}, \ldots, x_{2 l}=x+1, \ldots\right) \times\left(x_{1}+1, \ldots, x_{2 l+1}+1=x, \ldots\right) .
$$


LEMMA 2. Let

$\sigma=\left(b_{0}, \ldots, x, b_{2 r}, \ldots, b_{2 m}\right) \times\left(b_{1}+1, \ldots, x+1, b_{2 s-1}+1, \ldots, b_{2 m-1}+1\right)$

be a K-type such that $x<b_{2 r}$ and $x+1<b_{2 s-1}+1$. Assume that $r=s$ or $r=s+1$, and

$$
\sum_{\substack{j \geqslant r \\ i \geqslant s}}\left(b_{2 j}+b_{2 i-1}+1\right)=\sum_{\substack{j \geqslant r \\ i \geqslant s}}\left(x_{2 j}+x_{2 i-1}+1\right) .
$$

Let

$\sigma_{1}=\left(b_{0}, \ldots, \widehat{x}, b_{2 r}, \ldots, b_{2 m}\right) \times\left(b_{1}+1, \ldots, \widehat{x+1}, b_{2 s-1}+1, \ldots, b_{2 m-1}+1\right)$.

Then $[\sigma: X]=\left[\sigma_{1}, X_{1}\right]$.

Proof. Let $\mathcal{O}_{1}$ be the orbit corresponding to $X_{1}$. Recall that $X_{1}$ only contains $\mathrm{K}$-types that correspond to nilpotent orbits (in the Springer correspondence) that contain the orbit $\mathcal{O}_{1}$ in their closure. Thus it is enough to show that all $\operatorname{sgn} \otimes \sigma^{\prime}$ occuring in the restriction of $\sigma$ correspond to strictly smaller nilpotent orbits, except for the case of $\sigma_{1}$. For this, by Lemma 4.2, any $\sigma^{\prime}$ satisfies $b_{2 r-2}^{\prime}<x \leqslant b_{2 r}^{\prime}$ and $b_{2 s-3}^{\prime}+1<x+1 \leqslant b_{2 s-1}^{\prime}+1$. The $\mathrm{S}$-symbol is computed by forming $b_{2 i}^{\prime}+2 i$ and $b_{2 j-1}^{\prime}+2 j-2$ and reordering them in increasing order. Then all the entries with $i \geqslant 2 r$ and $j \geqslant 2 s-1$ are greater than $x+2 r-1$ (so they are permuted among themselves), while all other are strictly less than this number. If the sum of the entries greater than $x-2 r=1$ is strictly less than the corresponding sum for the $b$ 's, then the nilpotent orbit is strictly smaller. This involves the algorithm for computing the partition corresponding to the orbit from the symbol [9]. Thus to contribute to the multiplicity in $X$, we must have $b_{2 i}^{\prime}=b_{2 i}$ and $b_{2 j-1}^{\prime}=b_{2 j-1}$ for all $i \geqslant r$ and $j \geqslant s$. Then the claim follows from Lemma 1 of 4.2.

Thus the multiplicity of $\mu$ and $\mu^{\prime}$ in $X$ is the same as the multiplicity of $\mu_{1}$ and $\mu_{1}^{\prime}$ in $X_{1}$.

PROPOSITION. Let $\mu(\mathcal{W})$ be the lowest K-type of $\mathcal{W}$. Then

$$
\left[\mu^{\prime}: X(\mathcal{W}, \nu)\right]=[\mu(\mathcal{W}): \mathcal{W}]=1
$$

Proof. Apply the idea of the proof of Lemma 2. It is enough to show that the only K-type $\sigma^{\prime}$ that occurs in $\mu^{\prime} \otimes$ sgn and corresponds to a nilpotent containing $\mathcal{O}(\mathcal{W})$ in its closure is $\mu(\mathcal{W}) \otimes$ sgn. Suppose for example that $\mu$ is as in (4.2.14a) and $\mu^{\prime}$ as in (4.2.16a). The argument of the corollary implies that $\sigma^{\prime}$ must have the same $x_{2 i}^{\prime}$ for $i>k+1$ and the same $x_{2 j-1}^{\prime}+1$ for $j>k+1$ as $\mu$. Thus all the corresponding rows for the nilpotent that $\sigma^{\prime}$ is attached to coincide with the rows of the nilpotent $\mathcal{O}(\mathcal{W})$ attached to $\mathcal{W}$. Suppose $\sigma^{\prime} \neq \mu(\mathcal{W})$. Suppose that $x_{2 k+1}^{\prime}=E+1$ and $x_{2 k+2}^{\prime}=E-1$. This gives a nilpotent orbit which has the 
next two rows of size $2 E$. No such nilpotent will contain $\mathcal{O}(\mathcal{W})$ in its closure. The other choices for $\sigma^{\prime}$ are lowest $\mathrm{K}$-types for $\mathcal{O}(\mathcal{W})$, and the proof follows in this case.

The other cases follow by a similar calculation.

We conclude from the two lemmas and the proposition that $\mu$ and $\mu^{\prime}$ occur with multiplicity 1 in $X(\mathcal{W}, \nu)$. This means that they stay in the factor $X_{+}$for all $\nu$ and so their signatures are the same as for the corresponding $\mu_{1}$ and $\mu_{1}^{\prime}$. This is the same situation, but the corresponding $\mathcal{O}$ does not have the two $x_{i}=x$. The case when all $x_{i} \neq E$ are distinct is the case of $\mathcal{W}$ a discrete series.

We return to proving the claim that the K-types $\mu=\mu(\mathcal{O})$ and $\mu^{\prime}=\mu\left(\mathcal{O}^{\prime}\right)$ stay in the same factor, but now assuming that $\mathcal{W}$ is a discrete series.

Since all factors in the induced module correspond to nilpotent orbits containing $\mathcal{O}$ in their closure and there are no orbits between $\mathcal{O}$ and $\mathcal{O}^{\prime}$, the only way the two K-types cannot be in the same factors is if there is a factor in $X(\mathcal{W}, \nu)$ corresponding to $\mathcal{O}^{\prime}$. Essentially we can rule this out by infinitesimal character considerations. Suppose $L\left(\mathcal{W}^{\prime}, \nu^{\prime}\right)$ occurs in $X(\mathcal{W}, \nu)$ and has infinitesimal character $\chi^{\prime}$. Then $\chi^{\prime}$ must coincide with the infinitesimal character $\chi$ of $X(\mathcal{W}, \nu)$. In particular, the maximal orbits $\mathcal{O}_{\max }(\chi)$ and $\mathcal{O}_{\max }\left(\chi^{\prime}\right)$ from Section 2.3 must coincide. Suppose we are in case (4.2.13a). Then the corresponding infinitesimal characters which we write as

$$
\begin{aligned}
& (\ldots, 1, \ldots, E, 0, \ldots, E,-E+\nu, \ldots, E+\nu, \ldots) \\
& \quad\left(\ldots,-E-1+\nu_{1}, \ldots, E+\nu_{1},-E+\nu_{2}, \ldots, E-1+\nu_{2}, \ldots\right)
\end{aligned}
$$

must coincide. The contribution from the remaining $x_{i}$ 's must coincide because being distinct, the infinitesimal character cannot be deformed. Thus the entries written out in (4.2.23) must coincide. Reducibility considerations also force $\nu, \nu_{1}$ and $\nu_{2}$ to be integers. The infinitesimal characters coincide only if $\nu=1$. Since we are concerned with $\nu>1$, the claim follows in this case.

Consider case (4.2.9). If no $x_{i}=E+1$, then the proof is the same as the above. If there is an $x_{i}=E+1$, then

$$
\mathcal{O}^{\prime} \leftrightarrow(\ldots, 2 E, 2 E, 2 E+3,2 E+3, \ldots),
$$

and the possible infinitesimal characters are

$$
\begin{aligned}
& (\ldots, 0, \ldots, E,-E+\nu, \ldots, E+\nu, 1, \ldots, E+1, \ldots) \\
& \quad\left(\ldots,-E+\nu_{1}, \ldots, E-1+\nu_{1},-E-1+\nu_{2}, \ldots, E+1+\nu_{2}, \ldots\right) .
\end{aligned}
$$

As before, can ignore the other $x_{i}$ because being distinct the infinitesimal character cannot be deformed and so their contribution coincides for $\mathcal{O}_{\max }$ and $\mathcal{O}_{\max }^{\prime}$. Thus only case when the infinitesimal characters coincide for $\nu>1$, is at 
$\nu=2, \nu_{1}=\nu_{2}=1$. In this case $\nu_{2} \neq 0$. Then the irreducible module corresponding to $\mathcal{O}^{\prime}$ has two lowest K-types,

$$
\begin{aligned}
\mu^{\prime}= & \left(\ldots, x_{2 k}^{\prime}=E-1, x_{2 k+2}^{\prime}=E+1, \ldots\right) \times \\
& \times\left(\ldots, x_{2 k-1}^{\prime}+1=E+1, x_{2 k+1}^{\prime}=E+2, \ldots\right) \otimes \mathrm{sgn}, \\
\mu^{\prime \prime}= & \left(\ldots, x_{2 k}^{\prime}=E-1, x_{2 k}^{\prime}=E, \ldots\right) \times \\
& \times\left(\ldots, x_{2 k-1}^{\prime}+1=E+1, x_{2 k+1}^{\prime}=E+3, \ldots\right) \otimes \mathrm{sgn}
\end{aligned}
$$

or

$$
\begin{aligned}
\mu^{\prime}= & \left(\ldots, x_{2 k}^{\prime}=E, x_{2 k+2}^{\prime}=E+1, \ldots\right) \times \\
& \times\left(\ldots, x_{2 k+1}^{\prime}+1=E, x_{2 k+3}^{\prime}+1=E+2, \ldots\right) \otimes \mathrm{sgn}, \\
\mu^{\prime \prime}= & \left(\ldots, x_{2 k}^{\prime}=E, x_{2 k+2}^{\prime}=E+2, \ldots\right) \times \\
& \times\left(\ldots, x_{2 k+1}^{\prime}+1=E, x_{2 k+3}^{\prime}+1=E+1, \ldots\right) \otimes \mathrm{sgn} .
\end{aligned}
$$

It is enough to check that $\mu^{\prime \prime}$ does not occur in $X(\mathcal{W}, \nu)$. This follows by the same technique as Lemma 2 and Proposition of 4.2.

The other cases are similar but easier.

\subsection{THE INDUCTION STEP}

Suppose the representation is unitary. Label the $\nu$ 's in increasing order $\nu_{0}=0<$ $\nu_{1}<\cdots$. Assume the parameter is not unitarily induced from any Levi component of a proper parabolic subgroup. Suppose one of the strings, say $\left(f+\nu_{i}, \ldots, F+\right.$ $\nu_{i}$ ), does not satisfy (4.1.1). This means that $F+f>0$ or $F+f<-2$. Replace $\nu_{i}$ by $\nu$ and deform upwards in the first case, downwards in the second case until the first time the representation becomes reducible. First observe that the parameter does not cross any point where the parameter is unitarily induced irreducible from a proper Levi component. Indeed, suppose we are in the first case where we deform $\nu$ upwards to $1 / 2$ and no reducibility occurs. If the parameter were unitarily induced irreducible at any point in between, the string $(f+\nu, \ldots, F+\nu)$ must be involved. But this would imply $F+f=-2,-1,0$, which is not the case. For $1 / 2<\nu<1$, rewrite the string as

$$
\begin{aligned}
& (f+\nu, \ldots, F+\nu) \equiv\left(f^{\prime}+\nu^{\prime}, \ldots, F^{\prime}+\nu^{\prime}\right), \\
& \quad f^{\prime}=-F-1, \quad F^{\prime}=-f-1, \quad 0<\nu^{\prime}=1-\nu \leqslant \frac{1}{2} .
\end{aligned}
$$

The parameter $\nu^{\prime}$ is being deformed downward from $1 / 2$ to 0 . But then $f^{\prime}+F^{\prime}=$ $-f-F-2<-2$, and we are in the second case. The same argument applies. At $\nu^{\prime}=0$ we get a string

$$
(-F-1, \ldots,-f-1) \equiv(f+1, \ldots, F+1) .
$$


The only way the parameter stays irreducible, is if there is $x_{j}$ such that $x_{j}+1+\varepsilon<$ $f+1<F+1<x_{j+1}+1-\varepsilon$. This implies that the parameter is not unitarily induced irreducible. Write the string as $(f+1+\nu, \ldots, F+1+\nu)$ and continue $\nu$ upward. Say the first reducibility point occurs at a (deformed) $\nu$ such that $\nu \in \mathbb{Z}$. Then by induction all but the string $\left(f+\nu_{i}, \ldots, F+\nu_{i}\right)$ satisfy (4.1.1). We may as well replace the string being deformed by one of the form $\left(e+\nu^{\prime}, \ldots, E+\nu^{\prime}\right)$ of the form as in (3.3) with a $\nu^{\prime}>0$ very close to 0 , which does not satisfy (4.1.1) and also has the property that when $\nu^{\prime}$ is deformed to 0 , the parameter becomes reducible.

Say the first reducibility occurs at a $\nu$ such that $\nu-\nu_{j} \in \mathbb{Z}$ with $\nu_{j}>0$. There must be a string $\left(f^{\prime}+\nu_{j}, \ldots, F^{\prime}+\nu_{j}\right)$ such that if we deform both to some $\nu^{\prime}$ very close to $\nu_{j}$, the parameter becomes reducible. The factor containing sgn has the same strings as the original except possibly for the two involved in the reducibility, and corresponds to a strictly larger nilpotent orbit. By the induction hypothesis, all but these two strings are as in (4.1.1). If there are more strings besides these two, pick one and deform its $\nu$ until we reach a reducibility point. This involves at most one of the two bad strings. By induction the new parameter is unitary, so all but at most one string are as in (4.1.1). Suppose there are exactly two strings, neither satisfying (4.1.1). One of the strings is of the form

$$
\left(e+\nu_{i}, \ldots, E+\nu_{i}\right) \quad \text { satisfying } e>1+\varepsilon \text { or } E<-1-\varepsilon,
$$

the other $\left(f+\nu_{j}, \ldots, F+\nu_{j}\right)$. The deformation argument above allows us to assume that there is $\nu^{\prime}$ between $\nu_{i}$ and $\nu_{j}$ so that the induced representation becomes reducible when we deform $\nu_{i}$ and $\nu_{j}$ to $\nu^{\prime}$. We claim that either $E=$ $f-1$ or $e=F+1$; furthermore the string

$$
\begin{array}{ll}
\left(e+\nu^{\prime}, \ldots, F^{\prime}+\nu^{\prime}\right) & \text { if } E=f-1, \\
\left(f^{\prime}+\nu^{\prime}, \ldots, E+\nu^{\prime}\right) & \text { if } e=F+1
\end{array}
$$

is of type (4.1.1). Otherwise consider the parameter of the factor (at $\nu^{\prime}$ ) containing sgn. will have a string with lower endpoint $e+\nu^{\prime}$ if $e>1+\varepsilon$, or similarly a string with upper endpoint $E+\nu^{\prime}$ in case $E<-1-\varepsilon$. In both cases this contradicts the induction hypothesis: for example say we are in the second case of (4.3.4). Then $f+E=0,-1$ or -2 . Thus $f<0$. If $\nu_{j}>\nu^{\prime}>\nu_{i}$, deform $\nu_{j}$ upward to 1 . The parameter can only become reducible at $\nu_{j}=1-\nu_{i}$ or at $\nu_{j}=1$. It is certainly reducible at $\nu_{j}=1$, but this cannot be the first reducibility point. Suppose it were. The factor containing sgn has to be unitary. Because $f<0$, the parameter containing sgn corresponds to a strictly bigger nilpotent orbit. By induction, $(e+\nu, \ldots, E+\nu)$ has to satisfy (4.1.1), which is not the case. Thus the first reducibility point is $\nu_{j}=1-\nu_{i}$. At that point, the string is written $(-F-1+\nu, \ldots,-f-1+\nu)$. The same reasoning as before implies $-f=e$. This implies $F+f=e-1-e=-1$ contradicting the assumption. 
Suppose exactly one string $(e+\nu, \ldots, E+\nu)$ (with $0<\nu \leqslant 1 / 2)$ fails to satisfy (4.1.1) and the first reducibility occurs at a $\nu_{j}>0$. We check that we can place this string at $\nu_{1}$. If there are any smaller strings, we can deform their $\nu_{i}$ downward to 0 . The parameter must be either reducible or unitarily induced irreducible. The induction hypothesis applies to the factor containing sgn and in particular $(e+\nu, \ldots, E+\nu)$ must satisfy (4.1.1). Thus we may as well assume $\nu=\nu_{1}$.

Thus all strings but one at $\nu_{1}$ must satisfy (4.1.1). We can remove all strings having to do with Gl-complementary series by deformations as in the paragraphs between (C1) and (C4); no reducibility occurs due to the string at $\nu_{1}$. Indeed, for strings of type (4.1.1a) we deform to $1 / 2$, so away from $\nu_{1}$. For the others, we need not cross $\nu_{1}$ either. Note that because the other strings at $\nu_{1}$ must be nested, we can remove all the ones satisfying (4.1.1) as well. As a result, we are placed in the situation before (C4). If there is only one string left, we are done by (4.2). If there is more than one string left, there are exactly two, one of which satisfies (4.1.1b) or (4.1.1c). We write the string at $\nu_{1}$ as $\left(f+\nu_{1}, \ldots, F+\nu_{1}\right)$. Suppose it is $\left(-E+\nu_{2}, \ldots, E+\nu_{2}\right)$ with $\nu_{1} \leqslant \nu_{2}$. If we can deform $\nu_{2}$ to 0 without crossing any reducibility point, then the induction hypothesis implies that the string at $\nu_{1}$ must be of type (4.1.1). Furthermore it must be the case that $-E \leqslant f \leqslant F \leqslant E$ or $f \leqslant-E \leqslant E \leqslant F$.

In case the string at $\nu_{1}$ is as in (4.1.1a), we have two strings

$$
\left(-E^{\prime}-1+\nu_{1}, \ldots, E^{\prime}+\nu_{1}\right), \quad\left(-E+\nu_{2}, \ldots, E+\nu_{2}\right) .
$$

Then we can deform $\nu_{1}$ to $1 / 2$ without any reducibility. The ensuing representation is unitarily induced from Triv $\otimes *$ on $\mathrm{Gl}\left(2 E^{\prime}+2\right) G(*)$ and the conclusion of the theorem follows.

In case the string at $\nu_{1}$ is of the form (4.1.1b), we have two strings

$$
\left(-E^{\prime}+\nu_{1}, \ldots, E^{\prime}+\nu_{1}\right), \quad\left(-E+\nu_{2}, \ldots, E+\nu_{2}\right) .
$$

If $E^{\prime}=E$, we get a Gl-complementary series. If $E \neq E^{\prime}$, deform $\nu_{2}$ to 0 ; no reducibility occurs. By induction, the resulting representation must be unitary. It has a tempered parameter with two extra $x_{j}=E+\varepsilon$. For (C4) to be satisfied, there must be an $x_{i}=E^{\prime}+\varepsilon$ in the original parameter. Similarly deforming $\nu_{1}$ to 0 , we get that the tempered parameter has to have an $x_{i}=E+\varepsilon$. The induction step is completed in this case.

If the string at $\nu_{1}$ is as in (4.1.1c), the strings are

$$
\left(-E^{\prime}-1+\nu_{1}, \ldots, E^{\prime}-1+\nu_{1}\right), \quad\left(-E+\nu_{2}, \ldots, E+\nu_{2}\right) .
$$

If $E=E^{\prime}$, deforming $\nu_{2}$ to $1-\nu_{1}$ shows that the parameter is not unitary (cf. (C2)). In case $E \neq E^{\prime}$, we can deform $\nu_{1}$ to 1 . This adds two $x_{j}=E^{\prime}+\varepsilon$ to the tempered parameter. By the induction hypothesis, there must be an $x_{i}=E+\varepsilon$. Similarly deform $\nu_{2}$ to 0 to see that the tempered part of the parameter must 
have an $x_{i}=E^{\prime}+\varepsilon$. The induction step is complete in all cases when the string at $\nu_{2}$ is $\left(-E+\nu_{2}, \ldots, E+\nu_{2}\right)$ and no reducibility occurs when $\nu_{2}$ is deformed downward to 0 .

If on the other hand reducibility does occur when $\nu_{2}$ is deformed to 0 , we must have $f<-E \leqslant F<E$ or $-E<f \leqslant E<F$ (still with a string $\left.\left(-E+\nu_{2}, \ldots, E+\nu_{2}\right)\right)$. The induced module becomes reducible when we deform $\nu_{2}$ to $\nu_{1}$. The factor containing sgn has strings

$$
\begin{array}{lr}
\left(f+\nu_{1}, \ldots, E+\nu_{1}\right), & \left(-E+\nu_{1}, \ldots, F+\nu_{1}\right), \\
\left(-E+\nu_{1}, \ldots, F+\nu_{1}\right), & \left(f+\nu_{1}, \ldots, E+\nu_{1}\right) .
\end{array}
$$

We can deform the $\nu_{1}$ of either string to 0 . Thus each string must satisfy (A) and (B). This is the case only when the first string is

$$
\begin{aligned}
& \left(-E-2+\nu_{1}, \ldots, E-1+\nu_{1}\right), \\
& \left(-E-2+\nu_{1}, \ldots, E-2+\nu_{1}\right), \\
& \left(-E-1+\nu_{1}, \ldots, E-1+\nu_{1}\right), \text { or } \\
& \left(-E-1+\nu_{1}, \ldots, E-2+\nu_{1}\right),
\end{aligned}
$$

and the other string is $\left(-E+\nu_{2}, \ldots, E+\nu_{2}\right)$. Deform $\nu_{2}$ to $1-\nu_{1}$. In the first case the representation stays irreducible; we continue $\nu_{2}$ to 1 . This modifies the tempered part of the parameter, but in any case we obtain a parameter with a single string not of the form (4.1.1). The original parameter is not unitary. In the second case, the factor containing sgn has two strings $\left(-E-2+\nu_{1}, \ldots, E-1+\nu_{1}\right)$ and $\left(-E-1+\nu_{1}, \ldots, E-2+\nu_{1}\right)$. We can deform $\nu_{1}$ in the second one to 1 , to get a parameter with a single string not of the form (4.1.1); thus the original parameter is not unitary. In the third case, Lemma 4.1 shows that such a parameter cannot be unitary. The fourth case is similar to the first case.

The argument for when the other string is $\left(-E-1+\nu_{2}, \ldots, E-1+\nu_{2}\right)$ is the same. Since $0<\nu_{1}<\nu_{2} \leqslant 1 / 2 \leqslant 1-\nu_{2}<1-\nu_{1}<1$, we can deform $\nu_{2}$ upwards to $1-\nu_{2}$. Then we are back in the case of the string $\left(-E+\nu_{2}, \ldots, E+\nu_{2}\right)$. The proof of the induction step is complete.

\section{Acknowledgements}

The first author would like to thank the University of Paris VII for their hospitality during the spring of 1994 when some of these results were proved. We also thank the Institute for Advanced Study in Princeton for its hospitality and support during the fall of 1994. Both authors were supported in part by the National Science Foundation.

The results in this paper were presented at a workshop at the University of Twente in the winter of 1994. The first author would like to thank the organizers for the opportunity to give talks and publish this work in the proceedings of this conference. 


\section{References}

1. Borel, A.: Admissible representations of a semisimple group over a local field with fixed vectors under an Iwahori subgroup, Invent. Math. 35 (1976), 233-259.

2. Barbasch, D. and Moy, A.: A unitarity criterion for p-adic groups, Invent. Math. 98 (1989), 19-38.

3. Barbasch, D. and Moy, A.: Reduction to real infinitesimal character in affine Hecke algebras, J. Amer. Math. Soc. 6(3) (1993), 611-635.

4. Casselman, W.: A new nonunitarity argument for p-adic representations, J. Fac. Sci. Univ. Tokyo Sect. IA Math. 28(3) (1981), 907-928.

5. Carter, R.: Finite Groups of Lie Type, Wiley-Interscience, New York, 1985.

6. Kazhdan, D. and Lusztig, G.: Proof of the Deligne-Langlands conjecture for Hecke algebras, Invent. Math. 87 (1987), 153-215.

7. Lusztig, G.: Characters of Reductive Groups Over a Finite Field, Ann. Math. Studies 107, Princeton University Press.

8. Lusztig, G.: Affine Hecke algebras and their graded version, J. Amer. Math. Soc. 2 (1989), 599-635.

9. Lusztig, G.: Intersection cohomology complexes on a reductive group, Invent. Math. 75 (1984), $205-272$.

10. Lusztig, G.: Cuspidal local systems and graded algebras II, to appear in Proceedings of a Conference in Banff, 1994.

11. Tadic, M.: Classification of unitary representations in irreducible representations of general linear group (non-Archimedean case), Ann. Sci. Ec. Norm. Sup. 19 (1986), 335-382. 